\title{
Equivalences between blocks of cohomological Mackey algebras
}

\author{
Baptiste Rognerud
}

Received: 7 July 2014 / Accepted: 18 February 2015 / Published online: 5 March 2015

(C) Springer-Verlag Berlin Heidelberg 2015

\begin{abstract}
Let $G$ be a finite group and $(K, \mathcal{O}, k)$ be a $p$-modular system which is large enough. Let $R=\mathcal{O}$ or $k$. There is a bijection between the blocks of the group algebra $R G$ and the central primitive idempotents (the blocks) of the so-called cohomological Mackey algebra $\operatorname{co\mu }_{R}(G)$. Here, we introduce the notion of permeable derived equivalence and we prove that a permeable derived equivalence between two blocks of group algebras implies the existence of a derived equivalence between the corresponding blocks of cohomological Mackey algebras. In particular, in the context of Broué's abelian defect group conjecture, if two blocks are splendidly derived equivalent, then the corresponding blocks of cohomological Mackey algebras are derived equivalent.
\end{abstract}

Keywords Modular representation · Finite group · Mackey functor · Block theory

Mathematics Subject Classification $20 \mathrm{C} 05 \cdot 18 \mathrm{E} 30 \cdot 16 \mathrm{G} 10$

\section{Introduction}

The notion of Mackey functor, introduced by Green in [7], is a generalization of linear representations of a finite group $G$. A Mackey functor, for Green, is the data of a representation of $N_{G}(H)$ for every subgroup $H$ of $G$, together with relations between these representations. A couple of years later, Dress gave a completely different, but equivalent, definition using the formalism of categories. Twenty years later, Thévenaz and Webb introduced the Mackey algebra and proved that a Mackey functor is nothing but a module over this algebra.

A Mackey functor is cohomological if its restriction and induction maps behave like those of the cohomology of groups. The category consisting of cohomological Mackey functors is a full subcategory of the category of Mackey functors. This category is equivalent to the category of modules over the so-called cohomological Mackey algebra. Let $R$ be a

B. Rognerud $(\varangle)$

EPFL/SB/MATHGEOM/CTG, Station 8, 1015 Lausanne, Switzerland

e-mail: baptiste.rognerud@epfl.ch 
commutative ring. The cohomological Mackey algebras share a lot of properties of group algebras, for example $\operatorname{co\mu }_{R}(G)$ is $R$-free of finite rank and this rank is independent of the ring $R$. Moreover if $R$ is a field of characteristic which does not divide the order of $G$, then $\cos _{R}(G)$ is semi-simple. When $(K, \mathcal{O}, k)$ is a $p$-modular system, it is possible to define a decomposition theory for $\operatorname{co} \mu_{\mathcal{O}}(G)$, in particular the Cartan matrix of the cohomological Mackey algebra is symmetric. However there are some differences with group algebras: most of the time the determinant of the Cartan Matrix of $\cos _{k}(G)$ is zero. Moreover the cohomological Mackey algebra is not a symmetric algebra.

It has been noticed for a long time that there are deep links between the representation theory of finite groups and the theory of Mackey functors. Some objects of the first theory are much more natural when you see them via the Mackey functors' theory (e.g. p-permutation modules, Brauer quotient, ...). It is quite natural to think that this theory may be used in order to understand some open questions of the representation theory of finite group. The first attempt was about Alperin weight's conjecture. Jacques Thévenaz and Peter Webb proved that this conjecture is equivalent to a conjecture on Mackey functors. Here the arithmetic of the first conjecture is encoded in two Mackey functors.

In this paper, we propose to look at a Mackey functors version of Broué's abelian defect group conjecture.

Let $R=\mathcal{O}$ or $k$. In [16], Thévenaz and Webb proved that there is a bijection between the blocks of $R G$ and the primitive central idempotents of the so-called $p$-local Mackey algebra $\mu_{R}^{1}(G)$. In the proof, they remark that there is also a bijection between the blocks of $R G$ and the blocks of the cohomological Mackey algebra $\operatorname{co\mu }_{R}(G)$. Let us denote by $b \mapsto \iota(b)$ this bijection.

Using the Brauer correspondence, we have the following diagram: Let $b$ be a block of $R G$ with defect group $D$ and $b^{\prime}$ be its Brauer correspondent in $R N_{G}(D)$.

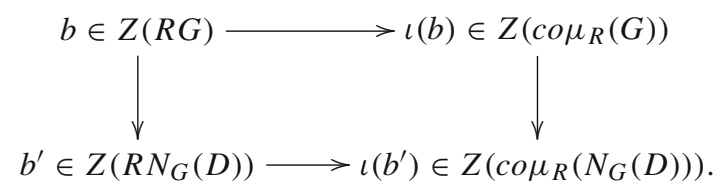

If $D$ is abelian, it is conjectured by Broué that the block algebras $R G b$ and $R N_{G}(D) b^{\prime}$ are deeply connected. It is a very natural question to ask if the same can happen for the corresponding Mackey algebras. However, we should notice that, since the cohomological Mackey algebra is not symmetric, the usual stable category is not triangulated, so we decided not to look at stable equivalences.

In this article we will focus on Morita equivalences and derived equivalences.

Question 1.1 (Bouc) Let $G$ be a finite group and $b$ be a block of $\mathcal{O} G$ with abelian defect group D. Let $b^{\prime}$ be the Brauer correspondant of $b$ in $\mathcal{O} N_{G}(D)$. Is there a derived equivalence $D^{b}\left(\operatorname{co\mu } \mu_{\mathcal{O}}(G) \iota(b)\right) \cong D^{b}\left(\operatorname{co} \mu_{\mathcal{O}}\left(N_{G}(D)\right) \iota\left(b^{\prime}\right)\right)$ ?

The main result of this paper is the following theorem which settles the question for the cohomological Mackey algebra in the case of a splendid equivalence [14]:

Theorem 1.2 Let $G$ and $H$ be two finite groups, let $b$ be a block of $R G$ and $c$ be a block of $R H$. If $R G b$ and $R H c$ are splendidly derived equivalent, then

$$
D^{b}\left(\operatorname{co\mu }_{R}(G) \iota(b)\right) \cong D^{b}\left(\operatorname{co\mu }_{R}(H) \iota(c)\right) .
$$


In the first part, we recall Yoshida's point of view on cohomological Mackey functors. There are several points of view on the notion of Mackey functors, so there are several points of view on the notion of cohomological Mackey functors. There are technical issues about the different versions of Yoshida's equivalence. A systematic use of the Burnside functor will clarify the situation. In the second part, we give an explicit isomorphism between the center of the group algebra and the center of the cohomological algebra. With this isomorphism we have a description of the blocks of the cohomological Mackey algebras. This decomposition is compatible with the block decomposition of the category of cohomological Mackey functors introduced by Thévenaz and Webb. With this description, we also prove a block version of Yoshida's theorem.

Using this block version of the Yoshida equivalence, we see that a so-called permeable Morita (resp. derived) equivalence between blocks of group algebras can be lifted to a Morita (resp. derived) equivalence between the corresponding blocks of cohomological Mackey algebras. For example splendid Morita equivalences, and splendid derived equivalences can be lifted. Even if the notion of permeable equivalence is very natural it seems to the author that it has not been considered yet. We investigate on the very basic properties of these equivalences. In particular, we show that in general, Morita equivalences are not permeable and we give an example of permeable Morita equivalence which is not splendid.

We give two applications of Theorem 1.2. The first one is a new point of view on Bouc's Theorem about the determinant of the Cartan matrices of the blocks of the cohomological Mackey algebras. He proved that this determinant is non zero if and only if the block is nilpotent with a cyclic defect group. The proof is based on a combinatorial approach and it may be surprising that nilpotent blocks appear in this context. We show that it is in fact very natural and comes from a structural reason. Finally we give an extremely naive application of Theorem 1.2 to representation of finite groups. If the Cartan matrices of two blocks $\operatorname{co\mu }_{R}(G) \iota(b)$ and $\operatorname{co\mu }_{R}(H) \iota(c)$ are not the same, then $R G b$ and $R H c$ are not splendidly (or permeable) Morita equivalent. This is a sufficient criterion for two blocks to not be splendidly Morita equivalent. This is particularly useful since it is possible to compute these matrices via an algorithm (in GAP4 e.g.). We give a particularly surprising example of nilpotent blocks with quaternion defect group, which was discovered by testing this algorithm.

Remark 1.3 The purpose of two first parts of this paper is to investigate the blocks of the cohomological Mackey algebra and to prove a block version of Yoshida's equivalence. If this proof involves rather technical discussions about Mackey functors, the result is not technical at all. Here, we do not assume the reader to be familiar with any deep result on Mackey functors. Still, if the reader is more interested by the link between splendid equivalences and equivalences between blocks of cohomological Mackey algebras, she/he might take Corollary 3.10 as a definition.

Notations: Let $R$ be a commutative ring with unit. We denote by $R$-Mod the category of (all) $R$-modules. We denote by $\operatorname{proj}(R)$ the category of finitely generated projective $R$-modules. Let $G$ be a finite group then a permutation projective $R G$-module is a direct summand of a permutation module. Let $p$ be a prime number. We denote by $(K, \mathcal{O}, k)$ a $p$-modular system, i.e. $\mathcal{O}$ is a complete discrete valuation ring with maximal ideal $\mathfrak{p}$, such that $\mathcal{O} / \mathfrak{p}=k$ is a field of characteristic $p$ and $\operatorname{Frac}(\mathcal{O})=K$ is a field of characteristic zero. If $R=\mathcal{O}$ or $k$, then the permutation projective $R G$-modules are called $p$-permutation modules. We denote by $G$-set the category of finite $G$-sets. If $H$ is a subgroup of $G$ then, we denote by $N_{G}(H)$ its normalizer in $G$. If $G$ is a finite group, the union of the transitive $G$-sets $G / H$ for all subgroups $H$ of $G$ is denoted by $\Omega_{G}$. That is 


$$
\Omega_{G}:=\bigsqcup_{H \leqslant G} G / H
$$

If $V$ is an $R$-module for a ring $R$ and $z \in Z(R)$, we denote by $m_{Z}(V)$ the morphism of $R$-modules induced by the multiplication by $z$ on $V$.

If $\mathcal{A}$ is an abelian category, we denote by $C^{-}(\mathcal{A})$ the category of right bounded complexes of $\mathcal{A}$, and by $C^{b}(\mathcal{A})$ the category of right and left bounded complexes of $\mathcal{A}$. We denote by $K^{-}(\mathcal{A})$ and $K^{b}(\mathcal{A})$ the corresponding homotopy categories, and finally by $D^{-}(A)$ and $D^{b}(A)$ the corresponding derived categories. Moreover, if $A$ is an $R$-algebra, we denote by $D^{s}(A)$ the derived category $D^{s}(A-M o d)$ for $s=b$ or $s=-$. Finally, if $X$ is an $A$-module (resp. a bounded complex of $A$-modules), we denote by $X^{*}$ the $R$-linear dual of $X$.

If $F: \mathcal{A} \rightarrow \mathcal{B}$ and $G: \mathcal{B} \rightarrow \mathcal{A}$ are two functors, we denote by $F \dashv G$ the fact that $F$ is a left adjoint of $G$.

N.B. We will denote by the same letter the block idempotents over the ring $\mathcal{O}$ and the field $k$.

\section{Yoshida's point of view on cohomological Mackey functors}

\subsection{Basic definitions}

For basic definitions of Mackey functors, we refer the reader to Section 2 of [16]. In this paper we will use Dress' point of view and Thévenaz-Webb's point of view. We will use Green's point of view only for the definition of cohomological Mackey functors since it is much more natural. Here, we just recall the definition of the Mackey algebra. Let $R$ be a commutative ring with unit.

Definition 2.1 The Mackey algebra $\mu_{R}(G)$ for $G$ over $R$ is the unital associative algebra with generators $t_{H}^{K}, r_{H}^{K}$ and $c_{g, H}$ for $H \leqslant K \leqslant G$ and $g \in G$, with the following relations:

- $\sum_{H \leqslant G} t_{H}^{H}=1_{\mu_{R}(G)}$.

- $t_{H}^{H}=r_{H}^{H}=c_{h, H}$ for $H \leqslant G$ and $h \in H$.

- $t_{K}^{L} t_{H}^{K}=t_{H}^{L}, r_{H}^{K} r_{K}^{L}=r_{H}^{L}$ for $H \subseteq K \subseteq L$.

- $c_{g^{\prime}, g} c_{g, H}=c_{g^{\prime} g, H}$, for $H \leqslant G$ and $g, g^{\prime} \in G$.

- $t_{g}^{g} K_{H} c_{g, H}=c_{g, K} t_{H}^{K}$ and $r_{g}^{g} K_{H}^{K} c_{g, K}=c_{g, H} r_{H}^{K}, H \leqslant K, g \in G$.

- $r_{L}^{H} t_{K}^{H}=\sum_{h \in[L \backslash H / K]} t_{L \cap h}^{L} c_{h, L^{h} \cap K} r_{L^{h} \cap K}^{K}$ for $L \leqslant H \geqslant K$.

- All the other products of generators are zero.

Definition 2.2 A Mackey functor for $G$ over $R$ is a left $\mu_{R}(G)$-module.

Proposition 2.3 The Mackey algebra is a free $R$-module, of finite rank independent of $R$. The set of elements $t_{K}^{H} c_{x, K^{x}} r_{K^{x}}^{L}$, where $H$ and $L$ are subgroups of $G$, where $x \in[H \backslash G / L]$, and $K$ is a subgroup of $H \cap{ }^{x} L$ up to $\left(H \cap{ }^{x} L\right)$-conjugacy, is an $R$-basis of $\mu_{R}(G)$.

Proof Section 3 of [16]. 
Now, let us recall the definition of the Burnside group of a finite $G$-set.

Definition 2.4 (2.4.1 [3]) If $X$ is a finite $G$-set, the category of $G$-sets over $X$ is the category with objects $(Y, \phi)$ where $Y$ is a finite $G$-set and $\phi$ is a morphism from $Y$ to $X$. A morphism $f$ from $(Y, \phi)$ to $(Z, \psi)$ is a morphism of $G$-sets $f: Y \rightarrow Z$ such that $\psi \circ f=\phi$.

The Burnside group of $X$, denoted by $B(X)$, is the Grothendieck group of the category of $G$-sets over $X$, for relations given by disjoint union. Moreover, we denote by $R B(X)$ the Burnside group after scalars extension. That is $R B(X)=R \otimes_{\mathbb{Z}} B(X)$.

Remark 2.5 If $X$ is a $G$-set, the Burnside group $R B\left(X^{2}\right)$ has a ring structure. A $G$-set $Z$ over $X \times X$ is the data of a $G$-set $Z$ and a map $(b \times a)$ from $Z$ to $X \times X$, denoted by $(X \stackrel{b}{\leftarrow} Y \stackrel{a}{\rightarrow} X)$. The product of (the isomorphism class of $)(X \stackrel{\alpha}{\leftarrow} Y \stackrel{\beta}{\rightarrow} X)$ and (the isomorphism class of) ( $X \stackrel{\gamma}{\leftarrow} Z \stackrel{\delta}{\rightarrow} X$ ) is given by (the isomorphism class of) the pullback along $\beta$ and $\gamma$.

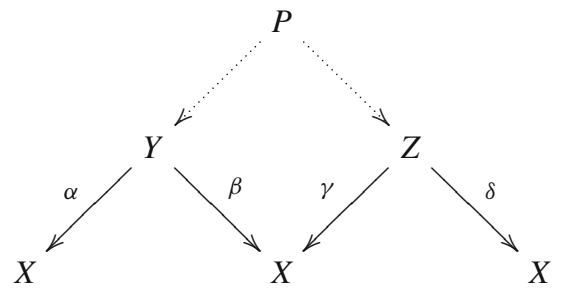

The identity of this ring is (the isomorphism class)

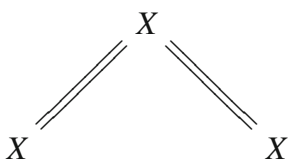

In the rest of the paper, we will denote by the same symbol a $G$-set over $X \times X$ and its isomorphism class in $R B(X \times X)$.

Let us recall that the Mackey algebra is isomorphic to a Burnside algebra:

Proposition 2.6 (Proposition 4.5.1 [3]) The Mackey algebra $\mu_{R}(G)$ is isomorphic to $R B\left(\Omega_{G}^{2}\right)$, where $\Omega_{G}=\sqcup_{L \leqslant G} G / L$.

Proof Let $H \leqslant K$ be two subgroups of $G$. We denote by $\pi_{H}^{K}$ the natural surjection from $G / H$ to $G / K$. If $g \in G$, then we denote by $\gamma_{H, g}$ the map from $G /{ }^{g} H$ to $G / H$ defined by $\gamma_{H, g}\left(x g \mathrm{Hg}^{-1}\right)=x g H$. The isomorphism $\beta$ is defined on the generators of $\mu_{R}(G)$ by:

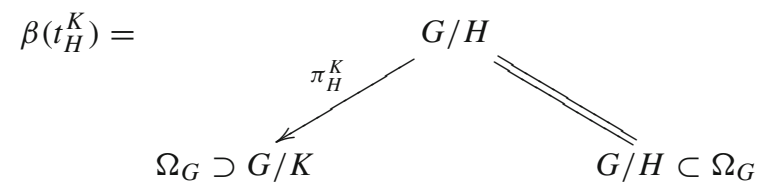




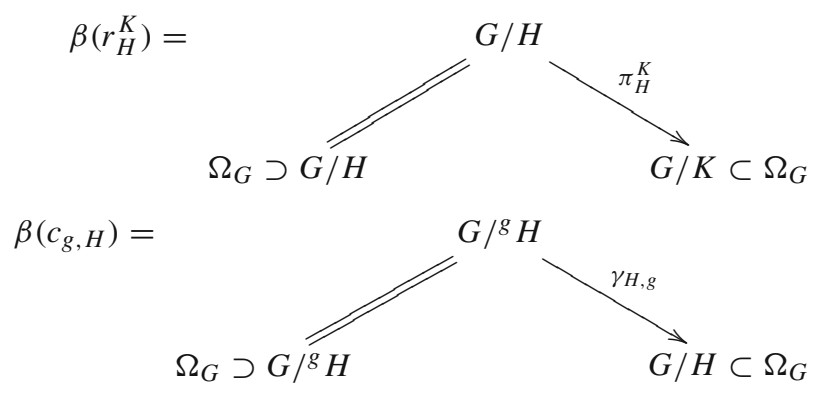

A Mackey functor $M$ for $G$, in the sense of Green, is cohomological if whenever $K \leqslant H \leqslant$ $G$, one has $t_{K}^{H} r_{K}^{H}=|H: K| I d_{M(H)}$. Let us denote by $\operatorname{Comack}_{R}(G)$ the full subcategory consisting of cohomological Mackey functors. The category $\operatorname{Comack}_{R}(G)$ is equivalent to the category of modules over the so-called cohomological Mackey algebra, denoted by $\cos _{R}(G)$. The cohomological Mackey algebra is the quotient of the Mackey algebra $\mu_{R}(G)$ by the ideal generated by the $t_{K}^{H} r_{K}^{H}-|H: K| t_{H}^{H}$ for $K \leqslant H \leqslant G$. If $x \in \mu_{R}(G)$, we denote by $\bar{x}$ its image in the quotient $\operatorname{co\mu }_{R}(G)$.

\subsection{Yoshida's equivalence}

In this section we recall Yoshida's theorem for cohomological Mackey functors. This theorem says that the category of cohomological Mackey functors for a group $G$ over a ring $R$, in the sense of Dress, is equivalent to the category of $R$-linear contravariant functors from the category of permutation projective modules to the category of $R$-modules. There are several points of view on the notion of Mackey functors, so for each of these points of view, we have a version of the Yoshida's theorem. In general it is not easy to move between these several versions. Since we will use in the next section an explicit version of Yoshida's theorem for the modules over the cohomological Mackey algebra and for Dress' point of view, we recall here how Yoshida's equivalence is defined. We believe that a systematic use of the Burnside functor will clarify the link between these different versions of Yoshida's Theorem.

Notations 2.7 Let $G$ be a finite group. Let $X, Y$ and $U$ be three finite $G$-sets. Let $a: U \rightarrow Y$ and $b: U \rightarrow X$ be two morphisms of $G$-sets. The $G$-set $(X \stackrel{b}{\leftarrow} U \stackrel{a}{\rightarrow} Y)$ over $X \times Y$ is denoted by $Z_{U, a, b}$. Let $H$ and $K$ be two subgroups of $G$. Let $x \in G$ and $L$ be a subgroup of $H \cap{ }^{x} K$. Then we denote by $Z_{H, K, L, X}$ the following $G$-set over $G / H \times G / K$ :

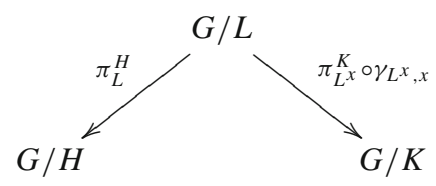

Here the maps $\pi_{L}^{H}$ and $\pi_{L^{x}}^{K}$ denote the canonical surjections from $G / L$ to $G / H$ and $G / L^{x}$ to $G / K$, respectively. Moreover, $\gamma_{L^{x}, x}$ is the map from $G / L$ to $G / L^{x}$ defined by $\gamma_{L^{x}, x}(g L)=$ $g x L^{x}$. We abuse notations by giving the same name of the previous $G$-set over $G H \times G / K$ and the corresponding $G$-set over $\Omega_{G} \times \Omega_{G}$ induced by the inclusion of $G / H$ and $G / K$ in $\Omega_{G}$. 
The main tool of this subsection is the so-called linearization Mackey functor:

Lemma 2.8 Let $X$ be a finite $G$-set. We set $\Pi(X)=R X$, that is the permutation $R G$ module with basis $X$. Let $f: X \rightarrow Y$ be a morphism of $G$-sets. Then we have a morphism of $R G$-modules $\Pi^{*}(f): R Y \rightarrow R X$ defined as follows:

$$
\Pi^{*}(f)\left(\sum_{y \in Y} r_{y} y\right)=\sum_{x \in X} r_{f(x)} x .
$$

On the other direction, we have a morphism $\Pi_{*}(f): R X \rightarrow R Y$ defined as follows:

$$
\Pi_{*}(f)\left(\sum_{x \in X} r_{x} x\right)=\sum_{x \in X} r_{x} f(x) .
$$

The bivariant functor $\Pi$ is a (non commutative) 'Mackey functor' with values in the category $R G$-Mod, i.e., we have:

- The bivariant functor $\Pi$ is additive.

- If

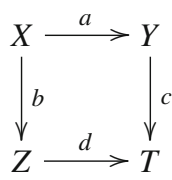

is a pullback diagram of $G$-sets, then

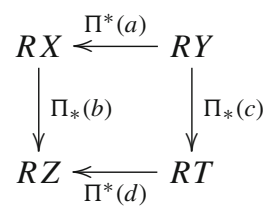

is a commutative diagram.

Proof Straightforward.

If the context is clear, we will simply denote by $f^{*}$ the morphism $\Pi^{*}(f)$ and by $f_{*}$ the morphism $\Pi_{*}(f)$.

Let $X, Y$ and $Z$ be finite $G$-sets. Let $R X, R Y$ and $R Z$ be the corresponding permutation $R G$-modules. Then, for $f \in H_{R} m_{R G}(R X, R Y)$ and $g \in H_{R} m_{R G}(R Y, R Z)$, we denote by $f \bullet g$ the usual composite $g \circ f$ from $R X$ to $R Z$.

Definition 2.9 Let $G$ be a finite group and $R$ be a commutative ring with unit. Then $E n d_{R G}\left(R \Omega_{G}\right)$, endowed with the product $\bullet$ defined above, is the Yoshida algebra for the group $G$ over the ring $R$.

Lemma 2.10 Let $X$ and $Y$ be two finite $G$-sets. Then there is a surjective map $p_{L}$, called the linear projection from $R B(X \times Y)$ to $\operatorname{Hom}_{R G}(R X, R Y)$, defined on a $G$-set over $X \times Y$ by:

$$
p_{L}(X \stackrel{b}{\leftarrow} Z \stackrel{a}{\rightarrow} Y)=a_{*} \circ b^{*}: R X \rightarrow R Z \rightarrow R Y,
$$

and extended by $R$-linearity to $R B(X \times Y)$. 
Proof By additivity, it is enough to check the result for two transitive $G$-sets. Let $H$ and $K$ be two subgroups of $G$. Let us suppose that $X=G / H$ and $Y=G / K$. For $x \in G$, let $Z_{H, K, H \cap x} K, x$ the $G$-set over $G / H \times G / K$ defined in Notations $2.7 \mathrm{Eq}$. (1). Then one can check that:

$$
p_{L}\left(Z_{H, K, H \cap \cap^{x} K, x}\right)(g H)=\sum_{h \in\left[H / H \cap{ }^{x} K\right]} g h x K .
$$

Moreover, up to isomorphism this $G$-set over $G / H \times G / K$ depends only on the double coset $H x K$. We will still denote by $Z_{H, K, H \cap x} K, x$ the image of this $G$-set in the Burnside group $R B(G / H \times G / K)$.

The result now follows from Lemma 3.1 of [18], which says that the set of morphisms $p_{L}\left(Z_{H, K, H \cap{ }^{x} K, x}\right)$ when $x$ runs through a set of representatives of the double cosets $H \backslash G / K$ is an $R$-basis of $\operatorname{Hom}_{R G}(R G / H, R G / K)$.

This linear projection is compatible with the composition of the morphisms in the following sense:

Lemma 2.11 Let $Z_{U, a, b}=(X \stackrel{b}{\leftarrow} U \stackrel{a}{\rightarrow} Y)$ be a $G$-set over $X \times Y$. Let $Z_{V, c, d}=(Y \stackrel{d}{\leftarrow}$ $V \stackrel{c}{\rightarrow} Z$ ) be a $G$-set over $Y \times Z$. Then

$$
p_{L}(X \stackrel{b}{\leftarrow} U \stackrel{a}{\rightarrow} Y) \bullet p_{L}(Y \stackrel{d}{\leftarrow} V \stackrel{c}{\rightarrow} Z)=p_{L}\left(Z_{U, a, b} \times Z_{V, c, d}\right),
$$

where the product $Z_{U, a, b} \times Z_{V, c, d}$ is as in Remark2.5, that is the pullback along the morphisms $a$ and $d$.

Proof This follows from the pullback property of the bivariant functor $\Pi=\left(\Pi^{*}, \Pi_{*}\right)$.

Theorem 2.12 (Yoshida's Theorem for cohomological Mackey algebra). Let $G$ be a finite group and $R$ be a commutative ring with unit. Then, there is an isomorphism of algebras $\phi: \operatorname{co\mu }_{R}(G) \rightarrow \operatorname{End}_{R G}\left(R \Omega_{G}\right)$, which makes the following diagram commutative:

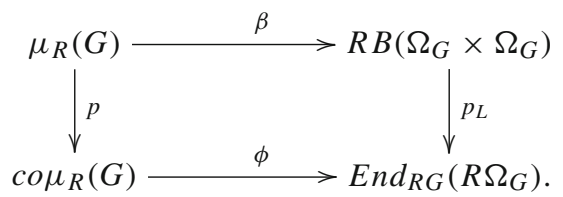

Here, the map $p: \mu_{R}(G) \rightarrow \operatorname{co\mu }_{R}(G)$ is the natural projection. The map $\beta$ is the isomorphism of Proposition 2.6, and $p_{L}$ is the linear projection of Lemma 2.10.

Proof We show that $\beta$ sends the kernel of $p$ into the kernel of $p_{L}$ and that $\beta^{-1}$ sends the kernel of $p_{L}$ into the kernel of $p$. Indeed, the kernel of $p$ is generated by the elements

$$
t_{H}^{K} r_{H}^{K}-|K: H| t_{K}^{K} \text {, for } H \leqslant K \leqslant G .
$$

This is sent to $Z_{K, K, H, 1}-|K: H| Z_{K, K, K, 1} \in R B\left(\Omega_{G} \times \Omega_{G}\right)$ by the morphism $\beta$. But $p_{L}\left(Z_{K, K, H, 1}\right)=|K: H| p_{L}\left(Z_{K, K, K, 1}\right)$. So,

$$
p_{L} \circ \beta\left(t_{H}^{K} r_{H}^{K}-|K: H| t_{k}^{k}\right)=0 .
$$

The other direction is more complicated. If an element $Z \in R B\left(\Omega_{G} \times \Omega_{G}\right)$ is such that $p_{L}(Z)=0$, then we can express $Z$ in the usual basis of $R B\left(\Omega_{G}^{2}\right)$, that is the basis induced by the isomorphism $\beta$ and the usual basis of the Mackey algebra (see Proposition 2.3, see also 
Proposition 3.2 of [16] for a direct proof). This basis is indexed by $H$ and $K$, two subgroups of $G$, an element $x$ of the set of representatives of the double cosets $H \backslash G / K$ and a subgroup $L$ of $H \cap{ }^{x} K$ (up to conjugacy). We denote by $I$ the set indexing this basis, and we denote by $Z_{H, K, L, x}$ the corresponding basis element. There are elements $\lambda_{H, K, L, x}$ of $R$ such that $Z=\sum_{I} \lambda_{H, K, L, x} Z_{H, K, L, x}$. Then $p_{L}(Z)=0$ if and only if for every $H, K$, we have:

$$
\sum_{L, x} \lambda_{H, K, L, x} p_{L}\left(Z_{H, K, L, x}\right)=0 .
$$

But, the 'Mackey functor' $\left(\Pi^{*}, \Pi_{*}\right)$ is cohomological in the following way:

$$
\begin{aligned}
p_{L}\left(Z_{H, K, L, x}\right)(g H) & =\sum_{h \in[H / L]} g h x K \\
& =\left|H \cap{ }^{x} K: L\right| \sum_{h \in\left[H / H \cap \cap^{x} K\right]} g h x K \\
& =\left|H \cap{ }^{x} K: L\right| p_{L}\left(Z_{H, K, H \cap{ }^{x} K, x}\right)(g H) .
\end{aligned}
$$

Moreover, the set of the maps $p_{L}\left(Z_{H, K, H \cap{ }^{x} K, x}\right)$ is, by Lemma 2.10, an $R$-basis of $\operatorname{Hom}_{R G}(R G / H, R G / K)$, so if $p_{L}(Z)=0$, we have, for $H$ and $K$ subgroups of $G$ and $x \in[H \backslash G / K]:$

$$
\sum_{L}\left|H \cap{ }^{x} K: L\right| \lambda_{H, K, L, x}=0 .
$$

Since in the cohomological Mackey algebra we have:

$$
p\left(t_{L}^{H} c_{x, L^{x}} r_{L^{x}}^{K}\right)=\left|H \cap{ }^{x} K: L\right| p\left(t_{H \cap{ }^{x} K}^{H} c_{x}, K \cap H^{x} r_{K \cap H^{x}}^{K}\right),
$$

then, if $p_{L}(Z)=0$, then we have

$$
\begin{aligned}
p \circ \beta^{-1}(Z) & =\sum_{H, K \leqslant G} \sum_{x \in[H \backslash G / K]} \sum_{L} \lambda_{H, K, L, x} p\left(t_{L}^{H} c_{x, L^{x}} r_{L^{x}}^{K}\right) \\
& =\sum_{H, K \leqslant G} \sum_{x \in[H \backslash G / K]}\left(\sum_{L} \lambda_{H, K, L, x}\left|H \cap{ }^{x} K: L\right|\right) p\left(t_{H \cap x}^{H} c_{x, K \cap H^{x}} r_{K \cap H^{x}}^{K}\right) \\
& =0 .
\end{aligned}
$$

In consequence $\beta$ and $\beta^{-1}$ induced mutually inverse isomorphisms between the kernel of $p$ and the kernel of $p_{L}$. By universal property of the cokernels, $\beta$ induces a morphism $\phi$ between $\operatorname{co\mu }_{R}(G)$ and $\operatorname{End}_{R G}\left(R \Omega_{G}\right)$ and $\beta^{-1}$ induces a morphism $\psi$ between $\operatorname{End}_{R G}\left(R \Omega_{G}\right)$ and $\operatorname{co\mu }_{R}(G)$. Now by Snake Lemma (e.g.) $\phi$ and $\psi$ are two mutually inverse isomorphisms of algebras.

As an immediate corollary, we have:

Corollary 2.13 Let $G$ be a finite group and $R$ be a commutative ring with unit. Then, the set of $\overline{t_{H \cap{ }^{x} K}^{H} c_{x}, K \cap H^{x} r_{K \cap H^{x}}^{K}}$, when $H$ and $K$ run through the subgroups of $G$ and $x$ runs through a set of representatives of double cosets $H \backslash G / K$ is an $R$-basis of $\operatorname{co} \mu_{R}(G)$.

Proof This follows from the fact that this set is the image of the $R$-basis of $\operatorname{End}_{R G}\left(R \Omega_{G}\right)$ of Lemma 2.10 introduced by Yoshida.

The linear-projection is a construction for the 'Mackey functor' $\left(\Pi^{*}, \Pi_{*}\right)$. As we saw in the proof of Theorem 2.12 the key point is the fact that this 'Mackey functor' is cohomological. 
We can copy this construction for an arbitrary cohomological Mackey functor. Let $X$ and $Y$ be two a finite $G$-sets. Let $M \in \operatorname{Comack}_{R}(G)$. We define an $R$-linear morphism $\tau_{M}$ between $R B(X \times Y)$ and $\operatorname{Hom}_{R}(M(Y), M(X))$ by sending a $G$-set $Z_{U, a, b}$ over $X \times Y$ to an $R$-linear morphism $\tau_{M}\left(Z_{U, a, b}\right)$ from $M(Y)$ to $M(X)$ which is defined by $\tau_{M}\left(Z_{U, a, b}\right)(m)=$ $M_{*}(b) M^{*}(a)(m)$ for $m \in M(X)$. We extend this definition by $R$-linearity to the elements of $R B(X \times Y)$. As in Lemma 2.11, this construction is compatible with the composition between the Burnside groups.

Lemma 2.14 Let $X$ and $Y$ be two finite $G$-sets. Let $M$ be a cohomological Mackey functor. If $Z \in R B(Y \times X)$ is in the kernel of the linear projection, then $\tau_{M}(Z)=0$.

Proof Using the fact that Mackey functors are additive, it is enough to check it for transitive $G$-sets. Let $H$ and $K$ be two subgroups of $G$. Let $Z \in R B(G / K \times G / H)$ such that $p_{L}(Z)=0$. Now the set of the $Z_{K, H, L, x}$ where $x$ runs through a set of representatives of the double cosets $K \backslash G / H$ and $L$ runs the through a set of representatives of the conjugacy classes of subgroups of $K \cap{ }^{x} H$ is an $R$-basis of $R B(G / K \times G / H)$ (Lemma 2.1 [16]). Since $M$ is a cohomological Mackey functor, we have

$$
\tau_{M}\left(Z_{K, H, L, x}\right)=\left|K \cap{ }^{x} H: L\right| \tau_{M}\left(Z_{K, H, K \cap{ }^{x} H, x}\right) .
$$

The result follows from the description of the kernel of $p_{L}$ in proof of Theorem 2.12

In conclusion, if $f \in \operatorname{Hom}_{R G}(R X, R Y)$ is a morphism of $R G$-modules between the permutations modules $R X$ and $R Y$, then we can define a morphism of $R$-modules from $M(Y)$ to $M(X)$ by sending $m \in M(Y)$ to $\tau_{M}(Z(f))(m)$ where $Z(f)$ is an element of $R B(X \times Y)$ such that $p_{L}(Z(f))=f$. Since it does not depend on the choice of $Z(f)$ in the pre-image of $f$, we simply denote by $\tau_{M}(f)$ this morphism.

Theorem 2.15 (Yoshida's Theorem for cohomological Mackey functors). Let $G$ be a finite group and $R$ be a commutative ring with unit. We denote by $F u n_{R}(G)$ the category of $R$-linear contravariant functors from the category of finitely generated permutation $R G$-modules to the category of $R$-modules. Then

$$
\operatorname{Comack}_{R}(G) \cong \operatorname{Fun}_{R}(G) .
$$

Sketch of proof. The existence of an equivalence between these two categories is a standard piece of category theory. But here we need an explicit equivalence, which can be constructed as follows: There is a Yoneda functor $Y$ from $\operatorname{Comack}_{R}(G)$ to $F u n_{R}(G)$. More precisely, if $M$ is a cohomological Mackey functor, then $Y(M)$ is defined by:

$$
Y(M)=\operatorname{Hom}_{\operatorname{Comack}_{R}(G)}(-, M) \circ F P_{-},
$$

where $F P_{-}$is the functor from the category of permutation $R G$-modules to the category of cohomological Mackey functors sending the $R G$-module $V$ to the fixed point functor $F P_{V}$. Here $F P_{V}$ is the Mackey functor defined by

$$
\operatorname{Hom}_{R G}(-, V) \circ \Pi \text {. }
$$

That is $F P_{V}(X)=\operatorname{Hom}_{R G}(\Pi(X), V)$ for a finite $G$-set $X$.

On the other hand, if $F \in F u n_{R}(G)$, then $\Gamma$ is defined by: $\Gamma(F)=F \circ \Pi$.

Let us recall the units and co-units of the two pairs of adjoint functors $\Gamma \dashv Y$ and $Y \dashv \Gamma$. 
- For the adjunction $\Gamma \dashv Y$ we have: let $F$ be a functor of $F u n_{R}(G)$. The unit $\delta$ of this adjunction is the natural transformation defined by: let $V=R X$ be a permutation $R G$-module and $u \in F(R X)$. Let $Z$ be a finite $G$-set. Then,

$$
\begin{aligned}
\delta_{F}(V)(u)_{Z}: \operatorname{Hom}_{R G}(R Z, R X) & \rightarrow F(R Z) \\
\alpha & \mapsto F(\alpha)(u) .
\end{aligned}
$$

Let $M$ be a cohomological Mackey functor. The co-unit of this adjunction is the map $\epsilon_{M}: \Gamma \circ Y(M) \rightarrow M$ defined by: let $X$ be a finite $G$-set. Then,

$$
\begin{aligned}
\epsilon_{M}(X): \operatorname{Hom}_{\operatorname{Comack}(G)}\left(F P_{R X}, M\right) \rightarrow M(X) \\
\alpha \mapsto \alpha_{X}\left(I_{R X}\right) .
\end{aligned}
$$

- For the second adjunction $Y \dashv \Gamma$, we have: let $F$ be a functor of $F u n_{R}(G)$ and let $M$ be a cohomological Mackey functor. Then the co-unit $\epsilon^{\prime}$ of this adjunction is defined as follows. Let $X$ be a finite $G$-set. Then:

$$
\begin{gathered}
\epsilon_{F}^{\prime}(X): \operatorname{Hom}_{\text {Oomack }_{R}(G)}\left(F P_{R X}, \Gamma(F)\right) \rightarrow F(R X) \\
\phi \mapsto \phi_{X}\left(I_{R X}\right) .
\end{gathered}
$$

For the unit it is a bit more complicated. Let $X$ and $Y$ be two finite $G$-sets. Let $m \in M(X)$. Let $f \in \operatorname{Hom}_{R G}(R Y, R X)$. Then by Lemma 2.10, the linear projection is surjective, so there exists $Z(f) \in R B(Y \times X)$ such that $p_{L}(Z(f))=f$. The unit of this adjunction is:

$$
\delta_{M}^{\prime}(X): M(X) \rightarrow \text { Hom }_{\text {Oomack }_{R}(G)}\left(F P_{R X}, M\right) .
$$

If $m \in M(X)$, then $\delta_{M}^{\prime}(X)(m)$ is the morphism of Mackey functors sending $f \in$ $F P_{R X}(Y)=\operatorname{Hom}_{R G}(R Y, R X)$ to $\tau_{M}(Z(f))(m) \in M(Y)$, where $\tau_{M}$ is defined above Lemma 2.14. Since $M$ is a cohomological Mackey functor, the map $\delta^{\prime}$ does not depend on the choice of $Z(f)$ so the co-unit is well defined.

Let us denote by $\operatorname{perm}_{R}(G)$ the full subcategory of $R G$-Mod consisting of the finitely generated permutation $R G$-modules.

Lemma 2.16 The idempotent completion of $\operatorname{perm}_{R}(G)$ is equivalent to the category of finitely generated permutation projective $R G$-modules.

Proof Let us denote temporarily by $\mathcal{A}$ the category of permutation projective $R G$-modules. Let $\operatorname{perm}_{R}^{+}(G)$ be the idempotent completion of $\operatorname{perm}_{R}(G)$.

The objects of this category are the pairs $(V, \pi)$ where $V$ is a permutation module and $\pi \in \operatorname{Hom}_{\operatorname{perm}_{R}(G)}(V, V)$ an idempotent. There is a natural functor $F$ from $\operatorname{perm}_{R}^{+}(G)$ to $\mathcal{A}$ defined by $F(V, \pi)=\pi(V)$. This functor is dense and fully faithful.

We denote by $\operatorname{perm}_{R}^{+}(G)$ the category of finitely generated permutation projective $R G$ modules and by $F u n_{R}^{+}(G)$ the category consisting of contravariant functors from $\operatorname{perm}_{R}^{+}(G)$ to $R$-Mod. By general properties of the idempotent completion ([1] Example 8.7.8 p. 97), the categories $F u n_{R}^{+}(G)$ and $F u n_{R}(G)$ are equivalent. So we have:

$$
\operatorname{Comack}_{R}(G) \cong \operatorname{Fun}_{R}^{+}(G) .
$$

We still denote by $Y \dashv \Gamma$ the equivalence after idempotent completion. 


\section{The center of the cohomological Mackey algebra}

Definition 3.1 Let $\mathcal{C}$ be a (small) additive category. The center of $\mathcal{C}$, denoted by $Z(\mathcal{C})$, is the endomorphism ring of the identity functor $I d_{\mathcal{C}}$ of the category $\mathcal{C}$.

It is well known that the definition of the center of a category is functorial with respect to equivalences of categories. Since we were not able to find a reference for this fact, we sketch the proof.

Lemma 3.2 Let $\mathcal{C}$ and $\mathcal{D}$ be two additive categories. Let $F \dashv G$ be an equivalence between $\mathcal{C}$ and $\mathcal{D}$. Then $F$ and $G$ induce mutually inverse isomorphisms between $Z(\mathcal{C})$ and $Z(\mathcal{D})$.

Proof We denote by $\delta$ (resp. $\delta^{\prime}$ ) the unit of the adjunction $G \dashv F$ (resp. $F \dashv G$ ) and by $\epsilon$ (resp. $\epsilon^{\prime}$ ) the co-unit of the adjunction $G \dashv F$ (resp. $F \dashv G$ ), that is the following natural transformations:

$$
\begin{gathered}
\delta: I d \rightarrow F G \\
\epsilon: G F \rightarrow I d \\
\delta^{\prime}: I d \rightarrow G F \\
\epsilon^{\prime}: F G \rightarrow I d .
\end{gathered}
$$

Let $\eta$ be an endomorphism of $I d_{\mathcal{C}}$. Then, $f(\eta)$ is the natural transformation from the functor $I d_{\mathcal{D}}$ to himself defined as follows: if $D$ is an object of $\mathcal{D}$, then:

$$
f(\eta)_{D}=\epsilon_{D}^{\prime} \circ F\left(\eta_{G(D)}\right) \circ \delta_{D}: D \rightarrow F G(D) \rightarrow F G(D) \rightarrow D .
$$

Let $\gamma$ be an endomorphism of $I d_{\mathcal{D}}$. Then $g(\gamma)$ is the natural transformation defined as follows: if $C$ is an object of $\mathcal{C}$, then:

$$
g(\gamma)_{C}=\epsilon_{C} \circ G\left(\gamma_{G(C)}\right) \circ \delta_{C}^{\prime}: C \rightarrow G F(C) \rightarrow G F(C) \rightarrow C .
$$

Remark 3.3 The definition of the center of an additive category generalizes the usual definition of the center of a ring. More precisely, if $R$ is a ring, then the center of the category $R$-Mod is isomorphic to the center of the ring $R$ (see the proof of Proposition 2.2.7 [2]). In particular, even if $R$-Mod is not a small category its center is well defined.

Proposition 3.4 Let $G$ be a finite group and $R$ be a commutative ring with unit. Then, there is a ring isomorphism:

$$
\iota: Z(R G) \rightarrow Z\left(\operatorname{co\mu }_{R}(G)\right)
$$

defined by:

$$
\iota(z)=\sum_{H \leq G} \sum_{g \in[H \backslash G / H]}\left(\sum_{x \in H} \lambda_{g x}\right) \overline{t_{H \cap \cap^{g} H}^{H} c_{g}, H \cap H^{g} r_{H \cap H^{g}}^{H}},
$$

for $z=\sum_{x \in G} \lambda_{x} x \in Z(R G)$, where we denote by $\bar{x}$ the image of $x \in \mu_{R}(G)$ in the cohomological Mackey algebra.

Proof The existence of an isomorphism between $Z(R G)$ and $Z\left(\cos \mu_{R}(G)\right)$ is due to Bouc (Proposition 12.3.2 of [3]). It uses the point of view of Green Mackey functors. More precisely it is based on the fact that cohomological Mackey functors are modules over the Green 
functor $F P_{R}$ and the fact that the Yoshida algebra is isomorphic to $F P_{R}\left(\Omega_{G} \times \Omega_{G}\right)$.

Here, we give an elementary proof of this result, which allows us to specify an isomorphism. First we prove that

$$
Z(R G) \cong Z\left(\operatorname{End}_{R G}\left(R \Omega_{G}\right)\right) .
$$

Let $z \in Z(R G)$. Then the multiplication by $z$ on the $R G$-module $R \Omega_{G}$, denoted by $m_{z}\left(R \Omega_{G}\right)$ is an element of the center of $\operatorname{End}_{R G}\left(R \Omega_{G}\right)$.

Let $H$ be a subgroup of $G$. We denote by $i_{H}$ the injection from $R G / H$ to $R \Omega_{G}$ and $p_{H}$ the projection from $R \Omega_{G}$ to $R G / H$. So, $f=\sum_{H, K \leqslant G} i_{K} \circ f_{K, H} \circ p_{H}$ where $f_{K, H}$ is a morphism in $\operatorname{Hom}_{R G}(R G / H, R G / K)$.

If $f \in \operatorname{End}_{R G}\left(R \Omega_{G}\right)$ is a central element, then, we have $f \circ g=g \circ f$ for $g \in \operatorname{End}_{R G}\left(R \Omega_{G}\right)$. By taking $g=i_{H} \circ p_{H}$ we see that

$$
f=\sum_{H \leqslant G} i_{H} \circ f_{H, H} \circ p_{H}
$$

where $f_{H, H} \in Z\left(\operatorname{End}_{R G}(R G / H)\right)$. By taking $g=i_{H} \circ\left(\pi_{1}^{H}\right)_{*} \circ p_{1}$, where $\left(\pi_{1}^{H}\right)_{*}$ is the linearization of the canonical projection from $G / 1$ to $G / H$, then for $x \in R G / 1$, we have $f_{H, H}\left(\left(\pi_{1}^{H}\right)_{*}(x)\right)=\left(\pi_{1}^{H}\right)_{*}\left(f_{1,1}(x)\right)$. That is, if $x=\sum_{g \in[G / H]} \lambda_{g} g H \in R G / H$, we have:

$$
\begin{aligned}
f_{H, H}(x) & =\sum_{g \in[G / H]} \lambda_{g} f_{H, H}(g H) \\
& =\sum_{g \in[G / H]} \lambda_{g}\left(\pi_{1}^{H}\right)_{*}\left(g f_{1,1}(1)\right) .
\end{aligned}
$$

But $f_{1,1}$ is a central element of $\operatorname{End}_{R G}(R G)$. So we have $g f_{1,1}(1)=f_{1,1}(1) g$. And for $x \in R G / H$ as above, we have:

$$
f_{H, H}(x)=\sum_{g \in[G / H]} \lambda_{g} f_{1,1}(1) g H=f_{1,1}(1) \cdot x .
$$

So $f=\sum_{H \leqslant G} i_{H} \circ m_{f_{1,1}(1)}(R G / H) \circ p_{H}=m_{f_{1,1}(1)}\left(R \Omega_{G}\right)$. If $f \in Z\left(\operatorname{End}_{R G}\left(R \Omega_{G}\right)\right)$, then $z=f_{1,1}(1) \in Z(R G)$ and $m_{f_{1,1}(1)}\left(R \Omega_{G}\right)=f$. It is clear that $m_{z}\left(R \Omega_{G}\right)_{1,1}(1)=z$. This proves that $Z(R G)$ is isomorphic to $Z\left(\operatorname{End}_{R G}\left(R \Omega_{G}\right)\right)$.

Since $\operatorname{co\mu } \mu_{R}(G) \cong \operatorname{End}_{R G}\left(R \Omega_{G}\right)$, we have:

$$
Z(R G) \cong Z\left(\operatorname{End}_{R G}\left(R \Omega_{G}\right)\right) \cong Z\left(\operatorname{co\mu }_{R}(G)\right) .
$$

It remains to specify an isomorphism. If $z \in Z(R G)$, then $z$ is a linear combination of elements of $G$, that is:

$$
z=\sum_{x \in G} \lambda_{x} x
$$

where $\lambda_{x} \in R$ for $x \in G$. Then, we have to find a pre-image of the multiplication by $z$ on $R \Omega_{G}$ by the linear projection. Let $H$ be a subgroup of $G$. We will show that:

$$
\sum_{g \in[H \backslash G / H]}\left(\sum_{x \in H} \lambda_{g x}\right) Z_{H, H, H \cap{ }^{g} H, g},
$$

is a pre-image of the multiplication by $z$ on $R G / H$ by the linear-projection. Since $z \in Z(R G)$, then for $h \in H$, we have $\lambda_{h g}=\lambda_{g h}$. Moreover the $G$-set $Z_{H, H, H \cap{ }^{g} H, g}$ over $G / H \times G / H$ 
depends only on the double coset $H g H$. So the formula (3) does not depend on the choice of a set of representatives of the double cosets $H \backslash G / H$. If $y H \in G / H$, then we have:

$$
\begin{aligned}
& \sum_{g \in[H \backslash G / H]}\left(\sum_{x \in H} \lambda_{g x}\right) p_{L}\left(Z_{H, H, H \cap{ }^{g} H, g}\right)(y H) \\
= & \sum_{g \in[H \backslash G / H]}\left(\sum_{x \in H} \lambda_{g x}\right) \sum_{h \in\left[H / H \cap{ }^{g} H\right]} y h g H \\
= & \sum_{g \in[H \backslash G / H]} \sum_{h \in\left[H / H \cap{ }^{g} H\right]}\left(\sum_{x \in H} \lambda_{h^{-1} h g x}\right) y h g H
\end{aligned}
$$

Since $z \in Z(R G)$, we have $\lambda_{h^{-1} h g x}=\lambda_{h g x h^{-1}}$. So by taking the sum on $x h^{-1} \in H$ instead of $x \in H$, we have:

$$
\begin{aligned}
& \sum_{g \in[H \backslash G / H]}\left(\sum_{x \in H} \lambda_{g x}\right) p_{L}\left(Z_{H, H, H \cap{ }^{g} H, g}\right)(y H) \\
= & \sum_{g \in[H \backslash G / H]} \sum_{h \in\left[H / H \cap{ }^{g} H\right]}\left(\sum_{x \in H} \lambda_{h g x}\right) y h g H
\end{aligned}
$$

Now the set of the $h g H$ when $g$ runs through a set of representatives of the double cosets $H \backslash G / H$ and $h$ runs through a set of representatives of the left cosets $H / H \cap{ }^{g} H$ is in bijection with a set of representatives of the left cosets $G / H$. So we have:

$$
\begin{aligned}
\sum_{g \in[H \backslash G / H]}\left(\sum_{x \in H} \lambda_{g x}\right) p_{L}\left(Z_{H, H, H \cap{ }^{g} H, g}\right)(y H) & =\sum_{t \in[G / H]}\left(\sum_{x \in H} \lambda_{t x}\right) y t H \\
& =y\left(\sum_{t \in[G / H]}\left(\sum_{x \in H} \lambda_{t x}\right) t\right) H
\end{aligned}
$$

But $\sum_{g \in G} \lambda_{g} g H=\sum_{t \in[G / H]}\left(\sum_{x \in H} \lambda_{t x}\right) t H$. Finally, we have:

$$
\sum_{g \in[H \backslash G / H]}\left(\sum_{x \in H} \lambda_{g x}\right) p_{L}\left(Z_{H, H, H \cap{ }^{g} H, g}\right)(y H)=y z H=z y H .
$$

So we have:

$$
m_{z}\left(R \Omega_{G}\right)=\sum_{H \leqslant G} \sum_{g \in[H \backslash G / H]}\left(\sum_{x \in H} \lambda_{g x}\right) p_{L}\left(Z_{H, H, H \cap{ }^{g} H, g}\right) .
$$

By the isomorphism of Theorem 2.12, this endomorphism of $R \Omega_{G}$ is sent to:

$$
\sum_{H \leq G} \sum_{g \in[H \backslash G / H]}\left(\sum_{x \in H} \lambda_{g x}\right) \overline{t_{H \cap g}^{H}{ }^{H} c_{g}, H \cap H^{g}} r_{H \cap H^{g}}^{H}
$$

Since the map sending $z \in Z(R G)$ to $m_{Z}\left(R \Omega_{G}\right)$ is a ring isomorphism and the map sending $m_{z}\left(R \Omega_{G}\right) \in \operatorname{End}_{R G}\left(R \Omega_{G}\right)$ to the corresponding element in $\operatorname{co} \mu_{R}(G)$ is an isomorphism of algebras, the composite is a ring isomorphism. 
Let $M$ be a cohomological Mackey functor. Let $X$ be a finite $G$-set and let $z$ be an element of $Z(R G)$. Let $f:=m_{z}(R X)$ be the multiplication by $z$ on $R X$. By Lemma 2.10, the linear projection is surjective, so there is a $Z(f) \in R B(X \times X)$ such that $m_{z}(R X)=p_{L}(Z(f))$. Then let us defined $\rho(z)_{M}(X): M(X) \rightarrow M(X)$ by sending $m \in M(X)$ to $\tau_{M}(Z(f))(m)$, where $\tau$ is defined above Lemma 2.14.

Lemma 3.5 Let $\rho: Z(R G) \rightarrow Z\left(\operatorname{Comack}_{R}(G)\right)$ be the map defined above. Then,

1. If $z \in Z(R G)$, then $\rho(z)$ is a well defined element in the center of $\operatorname{Comack}_{R}(G)$.

2. $\rho$ is a ring homomorphism.

Proof Since $M$ is a cohomological Mackey functor, by Lemma 2.14 this map does not depend on the choice of a pre-image of $m_{z} \in E n d_{R G}(R X)$ by the linear-projection. It is also clear that $\rho(z)$ is an element of the center of $\operatorname{Comack}_{R}(G)$. Now, using the pullback property of $M$, it is easy to check that $\rho$ is a ring homomorphism.

Remark 3.6 Since the categories $\operatorname{Comack}_{R}(G)$ and $\operatorname{com}_{R}(G)$-Mod are equivalent, their centers are isomorphic. The reader familiar with the equivalence of categories between Dress' definition and Green's definition (or Thévenaz-Webb's definition) can see that the morphism $\rho$ is just the morphism induced by $\iota$ and the equivalence of categories between $\operatorname{Comack}_{R}(G)$ and $\operatorname{co\mu } \mu_{R}(G)-M o d$.

Lemma 3.7 Let $G$ be a finite group. Let $\eta: Z(R G) \rightarrow Z\left(F u n_{R}^{+}(G)\right)$ be the map defined as follows. Let $z \in Z(R G)$ and let $F \in F u n_{R}^{+}(G)$. Let $V$ be a permutation projective module. Let us denote by $m_{z}$ the endomorphism of the identity functor of $R G$-Mod corresponding to $z$. Then $\eta_{z}$ is the endomorphism of the identity functor of $F u n_{R}^{+}(G)$ defined by:

$$
\eta_{z}(F)_{V}=F\left(m_{z}(V)\right): F(V) \rightarrow F(V) .
$$

The map $\eta$ is an ring homomorphism.

Proof This is straightforward.

The Yoshida equivalence is compatible with the action of central idempotents:

Theorem 3.8 (Yoshida Equivalence, block version). There is a commutative diagram:

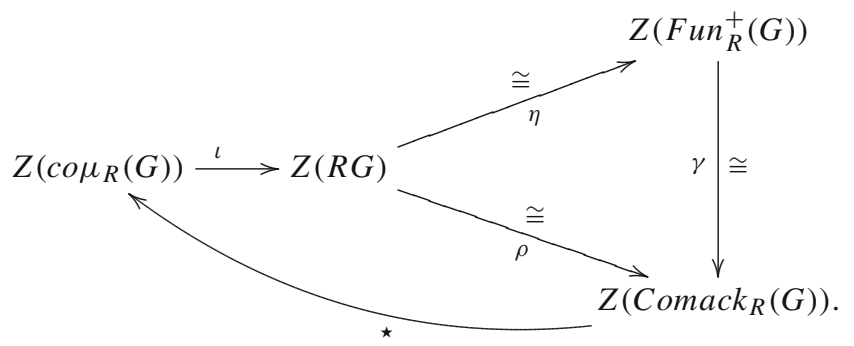

Here, the map $\gamma$ is the ring homomorphism induced by the functor $\Gamma$ as in Lemma 3.2. The arrow $\star$ is the map induced by the equivalence $\operatorname{Comack}_{R}(G)$ and $\cos _{R}(G)$-Mod (see Remark 3.6).

Let $1=e+f \in Z(R G)$ be a decomposition of 1 into an orthogonal sum of two idempotents. Then

$$
\operatorname{Comack}_{R}(G) \cong \rho(e)\left(\operatorname{Comack}_{R}(G)\right) \oplus \rho(f)\left(\operatorname{Comack}_{R}(G)\right) .
$$


and

$$
F u n_{R}^{+}(G)=\eta(e)\left(F u n_{R}^{+}(G)\right) \oplus \eta(f)\left(F u n_{R}^{+}(G)\right) .
$$

If $b=e$ or $f$, then $\rho(b)\left(\operatorname{Comack}_{R}(G)\right) \cong \eta(b)\left(F_{u n n_{R}^{+}}^{+}(G)\right)$.

Sketch of proof. Let $\sigma$ be a natural transformation of the identity functor of $\operatorname{Fun}_{R}^{+}(G)$, let $Y_{R G}$ be the Yoneda functor $\operatorname{Hom}_{R G}(-, R G)$, then $z_{\sigma}:=\left(\sigma_{Y_{R G}}(R G)\left(I d_{R G}\right)\right)(1)$ is an element of $Z(R G)$. One can check that the map sending $\sigma$ to $z_{\sigma}$ is the inverse isomorphism of $\eta$.

Let $M$ be a cohomological Mackey functor in the sense of Dress. Let $z \in Z(R G)$, we denote by $m_{z}$ the corresponding natural transformation in $Z(R G-M o d)$.

If $f \in Z\left(F u n_{R}^{+}(G)\right)$, then with the notations of Theorem 3.8, we have:

$$
\gamma(f)_{M}=\epsilon_{M} \circ \Gamma\left(f_{Y(M)}\right) \circ \delta_{M}^{\prime}: M \rightarrow \Gamma(Y(M)) \rightarrow \Gamma(Y(M)) \rightarrow M .
$$

So, if $X$ is a finite $G$-set, and if $m \in M(X)$, we have:

$$
\begin{aligned}
\gamma_{M}\left(\eta_{z}\right)(m) & =\delta_{M}^{\prime}(X) \circ\left(m_{z}(X)\right) \circ I d_{R X} \\
& =\delta_{M}^{\prime}(X)\left(m_{z}(X)\right)
\end{aligned}
$$

If $m_{z}(X)=p_{L}\left(\sum_{i=1}^{n} \lambda_{i} Z_{U_{i}, a_{i}, b_{i}}\right)$, where $Z_{U_{i}, a_{i}, b_{i}} \in R B(X \times X)$ and $\lambda_{i} \in R$, then we have:

$$
\gamma_{M}\left(\eta_{z}\right)(m)=\sum_{i=1}^{n} \lambda_{i} M_{*}\left(b_{i}\right) M^{*}\left(a_{i}\right)(m) .
$$

This is equal to $\rho(z)_{M}(X)$.

Let $R$ be $\mathcal{O}$ or $k$, where $\mathcal{O}$ is a complete discrete valuation ring and $k$ is the residue field. Let $1=b_{1}+b_{2}+\cdots+b_{s}$ be a decomposition of 1 into an orthogonal sum of central primitive idempotents of $R G$. This decomposition induces a decomposition of $\operatorname{Comack}_{R}(G)=\bigoplus_{i=1}^{s} \rho\left(b_{i}\right) \operatorname{Comack}_{R}(G)$ and $F_{u n}^{+}(G)=\bigoplus_{i=1}^{s} \eta\left(b_{i}\right) F u n_{R}^{+}(G)$. We have the following straightforward lemma:

Lemma 3.9 Let $b$ be a block idempotent of $R G$. The category $\eta(b)\left(F u n_{R}^{+}(G)\right)$ is equivalent to the category denoted by $F_{u n}^{+}(b)$, consisting of contravariant functors from perm $m_{R}^{+}(b)$ to $R$-Mod, where perm ${ }_{R}^{+}(b)$ is the category consisting of the finitely generated p-permutation $R G$-modules which are in the block $R G b$.

For a block $b$ of $R G$, we denote by $\operatorname{Comack}_{R}(b)$ the category $\rho(b) \operatorname{Comack}_{R}(G)$.

Corollary 3.10 Let $b$ be a block of $R G$. The we have:

$$
\operatorname{co\mu }_{R}(G) \iota(b)-M o d \cong \operatorname{Comack}_{R}(b) \cong \operatorname{Fun}_{R}^{+}(b) .
$$

Corollary 3.11 Let $P$ be a projective indecomposable cohomological Mackey functor. Then $P$ belongs to the block Comack $_{R}(b)$ if and only if $P(G / 1)$ is an indecomposable $p$ permutation module in the block $R G b$.

Proof Let $P$ be a cohomological Mackey functor. Let us recall that, with Dress' notation $P(G / 1)$ is an $R G$-module for the following action. Let $m \in P(G / 1)$ and $x \in G$. Then $x . m=P^{*}\left(\gamma_{1, x}\right)(m)$. The result follows from the fact that $(\rho(b) \cdot P)(G / 1)=b \cdot P(G / 1)$ and from Theorem 16.5 [16] which says that $P(G / 1)$ is a $p$-permutation module (it is also an easy consequence of Theorem 2.15). 
In the proof of Theorem 17.1 of [16], Thévenaz and Webb proved that the block of the category of the cohomological Mackey functors are in bijection with the block of $R G$. They defined the blocks of the category $\operatorname{Comack}_{R}(G)$ using non-split short exact sequences between simple cohomological Mackey functors. Thanks to Corollary 3.11 and Proposition 16.10 of [16] (in order to understand the projective cover of the simple cohomological Mackey functors), their block decomposition coincide with ours.

\section{Permeable Morita equivalences}

Let $R=\mathcal{O}$ or $k$ as above. With the version of Yoshida's equivalence of Corollary 3.10 it is not difficult to lift an equivalence between blocks of group algebras to an equivalence between the corresponding blocks of the cohomological Mackey algebras.

Definition 4.1 Let $G$ and $H$ be two finite groups, let $b$ be a block of $R G$, let $c$ be a block of $R H$. A permeable $R H c-R G b$-bimodule is a bimodule $X$ such that:

$\mathcal{P}: X \otimes_{R G b}-$ is a functor from $\operatorname{perm}_{R}^{+}(b)$ to $\operatorname{perm}_{R}^{+}(c)$.

Lemma 4.2 Let $G$ and $H$ be two finite groups, let $b$ be a block of $R G$, let $c$ be a block of $R H$. Let $X$ be a permeable $R H c$-RGb-bimodule. Then $X$ induces a functor, denoted by $\Phi_{X}:$ Comack $_{R}(c) \rightarrow$ Comack $_{R}(b)$ and defined in the proof. Moreover this functor sends an arbitrary fixed point functor to a fixed point functor.

Proof We use the equivalence $\operatorname{Comack}_{R}(b) \cong F u n_{R}^{+}(b)$ of Corollary 3.10. One can define a functor $L_{X}$ from $F u n_{R}^{+}(c)$ to $F u n_{R}^{+}(b)$ by $L_{X}(F)(V):=F\left(X \otimes_{R G b} V\right)$, for $F \in F u n_{R}^{+}(c)$ and $V \in \operatorname{perm}_{R}^{+}(b)$. We denote by $\Phi_{X}$ the composite functor:

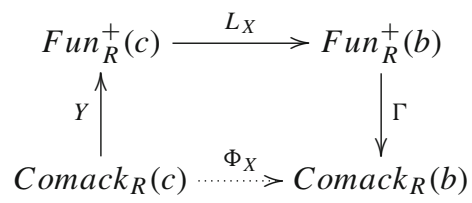

so if $V$ is a $R H c$-module, and $Z$ is a finite $G$-set, then

$$
\begin{aligned}
\Phi_{X}\left(F P_{V}\right)(Z) & =\Gamma\left(L_{X}\left(Y\left(F P_{V}\right)\right)\right)(Z) \\
& =Y\left(F P_{V}\right)\left(X \otimes_{R G b} R Z\right) \\
& \cong \operatorname{Hom}_{C_{\operatorname{mack}}(H)}\left(F P_{X \otimes_{R G b} R Z}, F P_{V}\right) \\
& \cong \operatorname{Hom}_{R H c}\left(X \otimes_{R G b} R Z, V\right) \\
& \cong \operatorname{Hom}_{R G b}\left(R Z, H o m_{R H c}(X, V)\right) \\
& \cong F P_{H_{R H c}(X, V)}(Z) .
\end{aligned}
$$

This isomorphism is functorial in $Z$, so $\Phi_{X}\left(F P_{V}\right)=F P_{H o m_{R H c}}(X, V)$.

Remark 4.3 This Lemma generalizes the construction defined by Bouc for permutation bimodules (see Section 3.12 [5]).

Definition 4.4 Let $G$ and $H$ be two finite groups, let $b$ be a block of $R G$ and $c$ be a block of $R H$. A permeable (Morita) equivalence between $R G b$ and $R H c$ is a Morita equivalence induced by a $R H c$-RGb-bimodule $X$ such that: 
1. $X \otimes_{R G b}-: R G b$-Mod $\rightarrow$ RHc-Mod is an equivalence of categories.

2. $X$ and $X^{*}:=\operatorname{Hom}_{R}(X, R)$ are two permeable bimodules.

Note that, since block algebras are symmetric $R$-algebras, the functor $X^{*} \otimes_{R H c}-$ is a quasi-inverse of $X \otimes_{R H b}-$.

Proposition 4.5 Let $G$ and $H$ be two finite groups, let $b$ be a block of $R G$ and $c$ be a block of $R H$. Let $X$ be a permeable equivalence between $R G b$ and $R H c$. Then $\operatorname{Comack}_{R}(b) \cong$ $\operatorname{Comack}_{R}(c)$.

Proof By Lemma 4.2, we have a functor $L_{X}: F u n_{R}^{+}(c) \rightarrow F u n_{R}^{+}(b)$, and a functor

$L_{X^{*}}: F_{n_{R}}^{+}(b) \rightarrow F u n_{R}^{+}(c)$. It is clear that these two functors are two quasi-inverse equivalences between $F u n_{R}^{+}(c)$ and $F u n_{R}^{+}(b)$.

Remark 4.6 One may ask if there exists permeable Morita equivalences. Let $G$ be a finite group, and $P$ be a Sylow $p$-subgroup of $G$ and $H$ be its normalizer. Let $b$ be a block of $k G$ and with defect group $P$ and let $c$ be the Brauer correspondent of this block in $N_{G}(P)$. If $k G b$-Mod is Morita equivalent to $k N_{G}(P) c$-Mod via a $p$-permutation bimodule (that is a 'splendid' Morita equivalence) then the two conditions are satisfied.

Remark 4.7 There exist $R G$ - $R H$-bimodules which are not $p$-permutation bimodules but which are permeable. The most radical example is for $G=H=C_{2}$ and $R=\overline{\mathbb{F}_{2}}$. Then, all the $R G$-modules are permutation modules. So every $R\left[C_{2} \times C_{2}\right]$-module induces a functor between $\operatorname{perm}_{R}(H)$ and $\operatorname{perm}_{R}(G)$, and there are infinitely many isomorphism classes of $R\left[C_{2} \times C_{2}\right]$-modules and only 5 isomorphism classes of permutation $R\left[C_{2} \times C_{2}\right]$-modules. Moreover, there are examples of Morita equivalences between blocks of group algebras which are not 'splendid' but which are permeable. The easiest example is probably for the self equivalences of $k C_{3}$ when $k=\mathbb{F}_{3}$. Indeed there are two permutation bimodules inducing a self-Morita equivalence of $k C_{3}$ and 6 isomorphism classes of self-Morita equivalences of $k C_{3}$. This follows from elementary results on the Picard group of a basic $k$-algebra and easy computations. Now all of these 6 equivalences are permeable.

\section{Derived equivalences between blocks of cohomological Mackey algebras}

Let $G$ and $H$ be two finite groups. Let $R=\mathcal{O}$ or $k$. Let $b$ be a block of $R G$ and $c$ be a block of $R H$. In this section, we prove that one can lift a derived equivalence between blocks of group algebras to a derived equivalence between the corresponding blocks of cohomological Mackey algebras as soon as this derived equivalence respects $p$-permutation modules.

Let $A=R G b$ and $B=R H c$, and ${ }_{A}$ Perm $_{B}$ be the category of permeable $A$ - $B$-bimodules. We want to produce a functor:

$$
C h\left({ }_{B} \operatorname{Perm}_{A}\right) \times C h\left(F u n_{R}^{+}(c)\right) \rightarrow C h\left(F u n_{R}^{+}(b)\right),
$$

which is obtained by 'pre-composition'. The goal is to show that this functor induces a functor between the appropriate homotopy categories.

Remark 5.1 The construction of this functor can be done in larger generality. Philosophically, If $A$ and $B$ are two rings with unit and $X$ is a complex of $A-B$-bimodules we can precompose a complex of additive functor in $F u n^{+}(A)$ by the complex $X$ in order to produce a complex of functors in $F u n^{+}(B)$. However there are set theoretical issues for the definition of the categories $\mathrm{Fun}^{+}(\mathrm{A})$ and $\mathrm{Fun}{ }^{+}(\mathrm{B})$ since the categories A-Mod and B-Mod are not skeletally 
small. In order to avoid these issues, one can look at $\mathcal{A}$ and $\mathcal{B}$ two skeletally small pre-additive full subcategories of $A-M o d$ and $B-M o d$, respectively. Then we have to look at the complexes of $A-B$-modules which map $\mathcal{B}$ to $\mathcal{A}$. But this will require complicate notations.

Since this section is rather technical, we fix the notations.

Notations - Let $X$ be an $R H c$-RGb-bimodule, then we denote by $t_{X}$ the functor from $R G b-M o d$ to $R H c$-Mod induced by the tensor product with $X$.

If $f: X \rightarrow Y$ is a morphism of $R G b$-RHc-bimodules, we denote by $\hat{f}$ the induced natural transformation between the functors $t_{X}$ and $t_{Y}$.

- Let $X$ be an $R H c$-RGb-bimodule such that $t_{X}$ induces a functor from $\operatorname{perm}_{R}^{+}(b)$ to $\operatorname{perm}_{R}^{+}(c)$. Let $F$ be a functor of $F u n_{R}^{+}(c)$, we can precompose the functor $F$ by the functor $t_{X}$, this gives a functor $F \circ t_{X}$ of the category $F u n_{R}^{+}(b)$. We will denote this functor by $F \tilde{X}$.

- Let $\left(F_{\bullet}, \eta_{\bullet}\right)$ be a complex of functors of $F u n_{R}^{+}(c)$. We choose to label the complex by decreasing order, that is $\eta_{i}$ is a natural transformation from the functor $F_{i}$ to the functor $F_{i-1}$.

- If $\left(X_{\bullet}, d_{\bullet}\right)$ is a complex (written in decreasing order) of permeable $R H c$ - $R G b$-bimodules, then $\left(\left(t_{X}\right)_{\bullet}, \hat{d}_{\bullet}\right)$ is the associated complex of functors from RGb-Mod to RHc-Mod.

Let $\left(F_{\bullet}, \eta_{\bullet}\right)$ be such a complex of functors and let $\left(X_{\bullet}, d_{\bullet}\right)$ be a complex of permeable $R H c$ - $R G b$-bimodules. Then we can precompose the complex $F_{\bullet}$ by the complex of functors $\left(t_{X}\right)$. This gives a double complex:

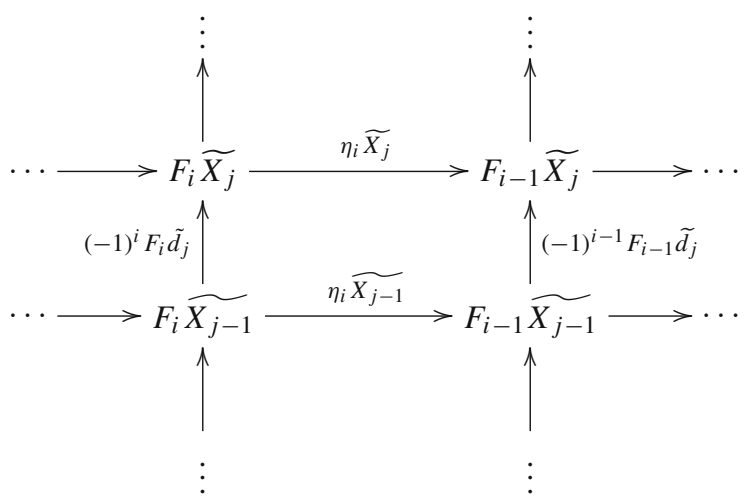

Here, we use the following notations:

1. Let $d: X \rightarrow Y$ be a map between two $R H c$-RGb-bimodules and let $F$ be a functor of the category $F u n_{R}^{+}(c)$. Then $F \tilde{d}$ is the natural transformation from $F \tilde{Y}$ to $F \tilde{X}$ defined by:

$$
F \tilde{d}(M)=F\left(d \otimes I d_{M}\right): F\left(Y \otimes_{R G b} M\right) \rightarrow F\left(X \otimes_{R G b} M\right),
$$

when $M$ is a $p$-permutation $R G b$-module.

2. Let $\eta$ be a natural transformation from $F$ to $C$, where $F$ and $C$ belong to $F u n_{R}^{+}(c)$. Let $X$ be a permeable $R G b$-RHc-bimodule. Then $\eta \tilde{X}$ is the natural transformation from $F \tilde{X}$ to $C \tilde{X}$ defined by: let $M$ be a $p$-permutation $R G b$-module. Then

$$
\eta \tilde{X}(M)=\eta\left(X \otimes_{R G b} M\right): F\left(X \otimes_{R G b} M\right) \rightarrow C\left(X \otimes_{R G b} M\right) .
$$


3. Note that since $\eta_{i}$ is a natural transformation from $F_{i}$ to $F_{i-1}$, we have:

$$
\eta_{i} \widetilde{X_{j}} \circ F_{i} \tilde{d}_{j}=F_{i-1} \tilde{d}_{j} \circ \eta_{i} \widetilde{X_{j-1}} .
$$

Let $\left(F_{\bullet}, \eta_{\bullet}\right)$ be a complex of functors which belong to $F u n_{R}^{+}(c)$. Let $\left(X_{\bullet}, d_{\bullet}\right)$ be a complex of permeable $R H c$ - $R G b$-bimodules. Then we denote by $\left(L_{X_{\bullet}}\left(F_{\bullet}\right), \delta_{\bullet}\right)$ the total complex of the double complex (5), that is:

$$
\left(L_{X_{\bullet}}\left(F_{\bullet}\right)\right)_{k}=\bigoplus_{i-j=k} F_{i} \widetilde{X_{j}},
$$

and the differential is given by the family of natural transformations $\delta_{k}$ defined by

$$
\delta_{k}=\bigoplus_{i-j=k}(-1)^{i} F_{i} \widetilde{d_{j+1}}+\eta_{i} \widetilde{X_{j}} .
$$

More explicitly, let $M$ be a $p$-permutation $R G b$-module.

Let $w=\left(w_{i, j}\right)_{i-j=k} \in \bigoplus_{i-j=k} F_{i}\left(X_{j} \otimes_{R G b} M\right)$. Then $\delta_{k}(M)=\bigoplus_{i-j=k} \delta_{i, j}(M)$, where:

$$
\delta_{i, j}(M)\left(w_{i, j}\right)=(-1)^{i} F_{i}\left(d_{j+1} \otimes I d_{M}\right)\left(w_{i, j}\right)+\eta_{i}\left(X_{j} \otimes_{R G b} M\right)\left(w_{i, j}\right) .
$$

Here, we use the notation $w_{i, j}$ which is the projection of $w$ on the component $F_{i}\left(X_{j} \otimes_{R G b} M\right)$.

\section{Lemma 5.2 With the previous notations,}

1. $\left(L_{X_{\bullet}}\left(F_{\bullet}\right), \delta_{\bullet}\right)$ is a complex.

2. If $X$ is a (left) bounded complex, then $F_{\bullet} \mapsto L_{X \bullet}\left(F_{\bullet}\right)$ is an additive functor from the category $\mathrm{Ch}^{-}\left(\mathrm{Fun}_{R}^{+}(c)\right)$ to the category $\mathrm{Ch}^{-}\left(\mathrm{Fun}_{R}^{+}(b)\right)$.

3. The functor $F_{\bullet} \mapsto L_{X}\left(F_{\bullet}\right)$ induces a triangulated functor between the corresponding homotopy categories.

Proof 1. The fact that the total complex of a double complex is a complex is standard.

2. Let $\left(F_{\bullet}, \eta_{\bullet}\right)$ and $\left(C_{\bullet}, \gamma_{\bullet}\right)$ be two complexes of functors which belong to $F_{u n}^{+}(c)$. Let $\phi=\left(\phi_{\bullet}\right)$ be a morphism from $\left(F_{\bullet}, \eta_{\bullet}\right)$ to $\left(C_{\bullet}, \gamma_{\bullet}\right)$. One may define a natural transformation $\Phi_{k}$ from $\left(L_{X_{\bullet}}\left(F_{\bullet}\right)\right)_{k}$ to $\left(L_{X_{\bullet}}\left(C_{\bullet}\right)\right)$ by: $\Phi_{k}:=\bigoplus_{i-j} \phi_{i} \widetilde{X_{j}}$, where $\phi_{i} \widetilde{X_{j}}$ is the natural transformation from $F_{i} \widetilde{X_{j}}$ to $C_{i} \widetilde{X_{j}}$ defined as follows: if $M$ is a $p$-permutation $R G b$-module, then

$$
\phi_{i} \widetilde{X_{j}}(M)=\phi\left(X_{j} \otimes_{R G b} M\right): F_{i}\left(X_{j} \otimes_{R G b} M\right) \rightarrow C_{i}\left(X_{j} \otimes_{R G b} M\right) .
$$

We have to check that $\left(\Phi_{k}\right)_{k \in \mathbb{Z}}$ is a morphism of complexes, i.e., we have to check that $\Phi$ commutes with the differentials.

We denote, here, by $\delta_{\bullet}$ the differential of $L_{X}\left(F_{\bullet}\right)$ and $\Delta_{\bullet}$ the differential of $L_{X_{\bullet}}\left(C_{\bullet}\right)$. Let $w \in \bigoplus_{i-j=k} F_{i}\left(X_{j} \otimes_{R G b} M\right)$. Then for $s-t=k-1$, we have:

$$
\begin{aligned}
\left(\left(\Phi_{k-1}(M) \circ \delta_{k}(M)\right)(w)\right)_{s, t}= & \phi_{s}\left(X_{t} \otimes_{R G b} M\right)\left(\delta_{k}(w)_{s, t}\right) \\
= & \phi_{s}\left(X_{t} \otimes_{R G b} M\right)\left(\eta_{s+1}\left(X_{t} \otimes M\right)\left(w_{s+1, t}\right)\right) \\
& +(-1)^{s} \phi_{s}\left(X_{t} \otimes_{R G b} M\right)\left(F_{s}\left(d_{t} \otimes I d_{M}\right)\left(w_{s, t-1}\right)\right) .
\end{aligned}
$$

On the other hand, we have:

$$
\begin{aligned}
\left(\Delta_{k}(M) \circ \Phi_{k}(M)(w)\right)_{s, t}= & \gamma_{s+1}\left(X_{t} \otimes M\right)\left(\phi_{s+1}\left(X_{t} \otimes_{R G b} M\right)\left(w_{s+1, t}\right)\right) \\
& +(-1)^{s} C_{s}\left(d_{s} \otimes I d_{M}\right)\left(\phi_{s}\left(X_{t-1} \otimes_{R G b} M\right)\left(w_{s, t-1}\right)\right) .
\end{aligned}
$$


So, the fact that $\Phi_{\bullet}$ is a morphism of complexes follows from the commutativity of these two diagrams:

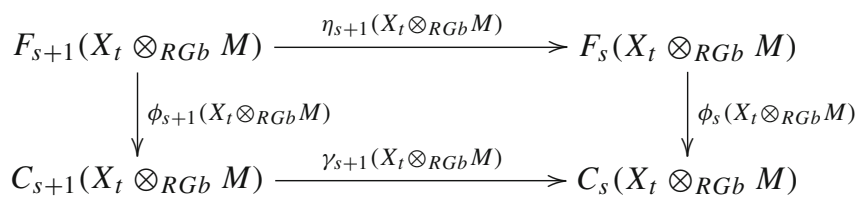

Here, the commutativity follows from the fact that $\phi_{\bullet}$ is a morphism of complexes.

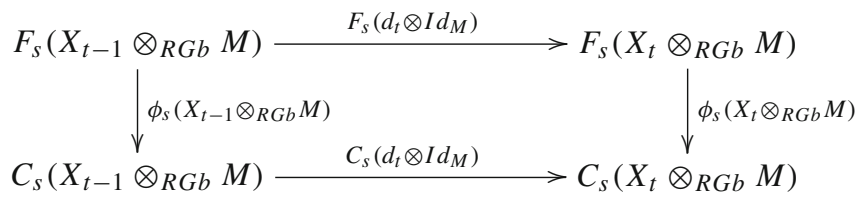

Here, the commutativity comes from the fact that $\phi_{s}$ is a natural transformation from $F_{S}$ to $C_{s}$. It is now clear that $L_{X_{\bullet}}$ is an additive functor, and we denote by $L_{X_{\bullet}}(\phi)$ the family of natural transformations $\Phi_{\bullet}$.

3. Since the functor $L_{X}$ is additive, it induces a functor between the corresponding homotopy categories. It remains to see that the functor $L_{X}$ is triangulated. It is clear that $L_{X_{\text {. }}}$ commutes with the shift, so we just have to check that it commutes with the mapping cone. Let $\left(F_{\bullet}, \eta_{\bullet}\right)$ and $\left(C_{\bullet}, \gamma_{\bullet}\right)$ be two complexes of functors which belong to $F_{u n}^{+}(c)$. Let $f$ be a morphism between these two complexes. We need to check that $L_{X_{\bullet}}($ cone $(f)) \cong \operatorname{cone}\left(L_{X_{\bullet}}(f)\right)$. We use the following notations:

- The differential of cone $(f)$ is denoted by $\beta$.

- The differential of $L_{X_{\bullet}}\left(F_{\bullet}\right)$ is denoted by $\delta$.

- The differential of $L_{X_{\bullet}}\left(C_{\bullet}\right)$ is denoted by $\Delta$.

- The differential of $L_{X}($ cone $(f))$ is denoted by $\partial$.

- The differential of cone $\left(L_{X_{\bullet}}(f)\right)$ is denoted by $D$.

Recall that ([17] Section 1.5) the mapping cone of $f$ is defined as follow:

$$
\operatorname{cone}(f)_{k}=F_{k-1} \oplus C_{k},
$$

and the differential is the natural transformation from cone $(f)_{k}$ to cone $(f)_{k-1}$ defined by the following diagram:

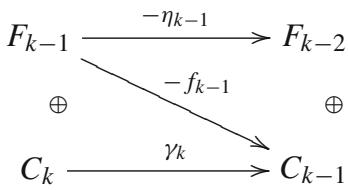


The differential $\partial_{k}$ from cone $\left(L_{X_{\bullet}}(f)\right)_{k}$ to cone $\left(L_{X_{\bullet}}(f)\right)_{k-1}$ is given in a similar way.

On the other hand,

$$
\begin{aligned}
L_{X_{\bullet}}(\text { cone }(f))_{k} & =\bigoplus_{i-j=k} \operatorname{cone}(f)_{i} \tilde{X}_{j} \\
& =\bigoplus_{i-j=k} F_{i-1} \tilde{X}_{j} \bigoplus_{i-j=k} C_{i} \tilde{X}_{j} \\
& =L_{X \bullet}(F)_{k-1} \oplus L_{X_{\bullet}}(C)_{k} .
\end{aligned}
$$

Let $M$ be a $p$-permutation $R G b$-module. Let $w \in \operatorname{cone}\left(L_{X_{\bullet}}(f)(M)\right)_{k}$, in order to compute the differential of this complex, we denote by $w^{F}$ the projection of the element $w$ on $\bigoplus_{i-j=k-1} F_{i}\left(X_{j} \otimes_{R G b} M\right)$, and $w^{C}$ the projection on $\bigoplus_{i-j=k} C_{i}\left(X_{j} \otimes_{R G b} M\right)$. Let $s$ and $t$ be integers such that $s-t=k-1$. Then the projection of $D_{k}(w)$ on $\operatorname{cone}(f)_{s, t}$ is:

$$
\begin{aligned}
\left(D_{k}(w)\right)_{s, t}= & \beta_{s+1} \widetilde{X_{t}}\left(w_{s+1, t}\right)+(-1)^{s} \operatorname{cone}(f)_{s} \widetilde{d}_{t}\left(w_{s, t-1}\right) \\
= & (-1)^{s} F_{s-1} \widetilde{d}_{t}\left(\left(w^{F}\right)_{s-1, t-1}\right)-\eta_{s}{\widetilde{X_{t}}}_{t}\left(\left(w^{F}\right)_{s, t}\right) \\
& +\gamma_{s+1} \widetilde{X_{t}}\left(\left(w^{C}\right)_{s+1, t}\right)+(-1)^{s} C_{s}\left(w_{s, t-1}^{C}\right)-f_{s} \widetilde{X_{t}}\left(\left(w^{F}\right)_{s, t}\right) \\
= & \left(-\delta_{k-1}\left(w^{F}\right)\right)_{s-1, t}+\left(\Delta_{k}\left(w^{C}\right)\right)_{s, t}-\left(L_{X}(f)\left(w^{F}\right)\right)_{s, t} \\
= & \partial_{k}(w)_{s, t} .
\end{aligned}
$$

So we have $L_{X \bullet}($ cone $(f)) \cong \operatorname{cone}\left(L_{X \bullet}(f)\right)$.

Lemma 5.3 Let $X_{\bullet}$ and $Y_{\bullet}$ be two bounded complexes of permeable $R H C$-RGb-bimodules.

Then:

1. The two functors $L_{X_{\bullet} \oplus Y_{\bullet}}$ and $L_{X} \oplus L_{Y_{\bullet}}$ are isomorphic as functors from $K^{-}\left(F u n_{R}^{+}(c)\right)$ to $K^{-}\left(F u n_{R}^{+}(b)\right)$.

2. If the complex $X_{\bullet}$ is contractible, then the functor $L_{X_{\bullet}}$ is contractible in the following sense: the complex $L_{X_{\bullet}}\left(F_{\bullet}\right)$ is (naturally in $F$ ) contractible for every complex $F_{\bullet}$ of functors which belong to $\mathrm{Fun}_{R}^{+}(\mathrm{c})$.

Proof 1 . Let $\left(F_{\bullet}, \eta_{\bullet}\right)$ and $\left(C_{\bullet}, \gamma_{\bullet}\right)$ be two complexes of functors which belong to $F u n_{R}^{+}(c)$. Let $f: F_{\bullet} \rightarrow C_{\bullet}$ be a morphism between these two complexes. It is clear that $L_{X_{\bullet} \oplus Y_{\bullet}}\left(F_{\bullet}\right) \cong L_{X_{\bullet}}\left(F_{\bullet}\right) \oplus L_{Y_{\bullet}}\left(F_{\bullet}\right)$. Let $M$ be a $p$-permutation $R G b$-module, let $j$ be an integer. We denote by $\zeta_{M, j}$ the composite:

$$
X_{j} \otimes_{R G b} M \rightarrow X_{j} \otimes_{R G b} M \oplus Y_{j} \otimes_{R G b} M \cong\left(X_{j} \oplus Y_{j}\right) \otimes_{R G b} M .
$$

The functoriality of the isomorphism follows from the fact that, for $i, j \in \mathbb{Z}$, the following diagrams (and the corresponding diagrams for the terms of $Y_{\bullet}$ ) are commutative:

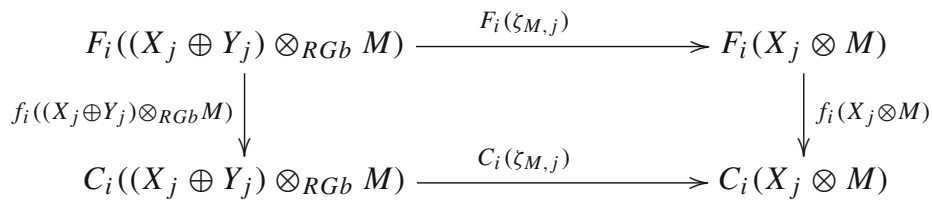


2. Let $X$. be a contractible two-sided bounded complex. That is, there is a family of maps $s=\left(s_{j}\right)_{j \in \mathbb{Z}}$, where $s_{j}$ is a map from $X_{j}$ to $X_{j+1}$, such that we have for $j \in \mathbb{Z}$ :

$$
I d_{X_{j}}=s_{j-1} d_{j}+d_{j+1} s_{j}
$$

Let $\left(F_{\bullet}, \eta_{\bullet}\right)$ be a complex of functors which belong to $F u n_{R}^{+}(c)$. Then one can defined a family $(F \widetilde{s})$ of natural transformations $(F \widetilde{s})_{k}$ from $L_{X_{\bullet}}\left(F_{\bullet}\right)_{k}$ to $L_{X_{\bullet}}\left(F_{\bullet}\right)_{k+1}$ as:

$$
(F \widetilde{s})_{k}=\bigoplus_{i-j=k}(-1)^{i} F_{i} \widetilde{s_{j-1}}
$$

where $F_{i} \widetilde{s_{j-1}}$ is the natural transformation defined as: let $M$ be a $p$-permutation $R G b$ module. Then

$$
F_{i} \widetilde{s_{j-1}}(M)=F_{i}\left(s_{j-1} \otimes_{R G b} I d_{M}\right): F_{i}\left(X_{j} \otimes_{R G b} M\right) \rightarrow F_{i}\left(X_{j-1} \otimes_{R G b} M\right) .
$$

Now, one can check that $I d_{L_{X_{\bullet}}\left(F_{\bullet}\right)}=\delta_{k+1} F \widetilde{s}_{k}+F \widetilde{s}_{k-1} \delta_{k}$. Moreover, this construction is functorial in $F$, so the functor $L_{X}$, is isomorphic to the zero functor from $K^{-}\left(F u n_{R}^{+}(c)\right)$ to $K^{-}\left(F u n_{R}^{+}(b)\right)$ when $X_{\bullet}$ is contractible.

Lemma 5.4 Let $G, H$ and $K$ be finite groups. Let $b$ be a block of $R G$, let $c$ be a block of $R H$ and let $d$ be a block of $R K$. Let $\left(X_{\bullet}, d_{\bullet}^{X}\right)$ be a bounded complex of permeable $R H b$ $R G$ c-bimodules. Let $\left(Y_{\bullet}, d_{\bullet}^{Y}\right)$ be a bounded complex of permeable RKd-RHc-bimodules. Then, we have an isomorphism of functors:

$$
L_{X} \circ L_{Y} \cong L_{Y \otimes_{R H c} X}
$$

Proof We use the following convention for the tensor product of complexes:

$$
Y_{\bullet} \otimes_{R H c} X_{\bullet}=\bigoplus_{i+j=k} Y_{i} \otimes_{R H c} X_{j}
$$

the differential, denoted by $D_{\bullet}$ is:

$$
D_{k}=\bigoplus_{i+j=k}\left((-1)^{i} I d_{Y_{i}} \otimes d_{j}^{X}+d_{i}^{Y} \otimes I d_{X_{j}}\right) .
$$

Let $M$ be a $p$-permutation $R G b$-module and let $k$ be an integer. Let $F_{\bullet}$ be a complex of functors which belong to $F u n_{R}^{+}(d)$. Since the functors $F_{i}$ are additive functors, and since $X$. and $Y_{\bullet}$ are bounded complex, it is clear that:

$$
L_{X \bullet} \circ L_{Y_{\bullet}}(F)(M)_{k} \cong L_{Y_{\bullet} \otimes_{R H c} X \cdot}(F)(M)_{k} .
$$


If we denote by $\Delta$ the differential of $L_{X \bullet} \circ L_{Y_{\bullet}}(F)$, by $\partial$ the differential of $L_{Y_{\bullet} \otimes_{R H c} X}(F)$ and by $\delta$ the differential of $L_{Y_{\bullet}}(F)$, we have:

$$
\begin{aligned}
\Delta_{k}(M)= & \bigoplus_{n \in \mathbb{Z}} \delta_{n}\left(X_{n-k} \otimes_{R G b} M\right)+(-1)^{n} L_{Y}(F)_{n}\left(d_{n-k+1}^{X} \otimes_{R G b} I d_{M}\right) \\
= & \bigoplus_{n \in \mathbb{Z}}\left(\bigoplus_{m \in \mathbb{Z}} \eta_{m}\left(Y_{m-n} \otimes_{R H c} X_{n-k} \otimes_{R G b} M\right)\right. \\
& +(-1)^{m} F_{m}\left(d_{m-n+1}^{Y} \otimes_{R H c} I d_{X_{n-k}} \otimes_{R G b} I d_{M}\right) \\
& +(-1)^{m}(-1)^{n-m} F_{m}\left(I d_{Y_{m-n}} \otimes_{R H c} d_{n-k+1}^{X} \otimes_{R G b} I d_{M}\right) \\
= & \bigoplus_{m \in \mathbb{Z}}\left(\eta_{m}\left(\left(Y_{\bullet} \otimes_{R H c} X_{\bullet}\right)_{m-k} \otimes_{R G b} M\right)\right. \\
& +(-1)^{m} F_{m}\left(\bigoplus_{m \in \mathbb{Z}} d_{m-n}^{Y} \otimes_{R H c} I d_{X_{m-k+1}} \otimes_{R G b} I d_{M}\right) \\
= & \left.(-1)^{m} F_{m}\left(\bigoplus_{m \in \mathbb{Z}}(-1)^{m-n} I d_{Y_{m-n}} \otimes_{R H c} d_{n-k+1}^{X} \otimes_{R G b} I d_{M}\right)\right) \\
= & \bigoplus_{m \in \mathbb{Z}}\left(\eta_{m}\left(\left(Y_{\bullet} \otimes_{R H c} X_{\bullet}\right)_{m-k} \otimes_{R G b} M\right)+(-1)^{m} F_{m}\left(D_{m-k+1} \otimes_{R G b} I d_{M}\right)\right) \\
= & \partial_{k}(M) .
\end{aligned}
$$

Since the isomorphism $L_{X} \circ L_{Y}(F) \cong L_{Y \otimes_{R H C} X}(F)$ basically involves only some isomorphisms of the form $F(V \oplus W) \cong F(V) \oplus F(W)$, for some $R K d$-modules, which are functorial in $F$, the isomorphism $L_{X} \circ L_{Y}(F) \cong L_{Y \otimes_{R H c} X}(F)$ is functorial in $F$.

Definition 5.5 Let $G$ and $H$ be two finite groups. Let $b$ be a block of $R G$ and $c$ be a block of $R H$. Then a permeable derived equivalence between $R G b$ and $R H c$ is a derived equivalence induced by a bounded complex $X$ of $R G b$-RHc-bimodules, which are projective as $R G b$ module and as $R H c$-module, such that:

- $X \otimes_{R H c} X^{*} \cong R G b$ in the homotopy category of $R G b$-bimodules. That is there exists a contractible complex $C$ of (permeable) $R G b$-bimodules such that

$$
X \otimes_{R H c} X^{*}=R G b \oplus C .
$$

- $X^{*} \otimes_{R G b} X \cong R H c$ in the homotopy category of $R H c$-bimodules. That is there exists a contractible complex $C^{\prime}$ of (permeable) $R H c$-bimodule such that

$$
X^{*} \otimes_{R G b} X=R H c \oplus C^{\prime} .
$$

- All the terms of the complexes $X$ and $X^{*}$ are permeable bimodules.

The complexes $X$ and $X^{*}$ are called permeable (two-sided) tilting complexes.

Remark 5.6 It is clear that a splendid derived equivalence (see [14]) is a permeable equivalence since all the terms of the tilting complex are $p$-permutation bimodules.

Lemma 5.7 Let $X_{\bullet}$ be a bounded complex which induces a permeable derived equivalence between $R H c$ and $R G b$. Then the functor $L_{X}$ induces a functor from $K^{b}\left(\operatorname{proj}\left(F u n_{R}^{+}(c)\right)\right)$ to $K^{b}\left(\operatorname{proj}\left(F u n_{R}^{+}(b)\right)\right)$. 
Proof The finitely generated projective objects of the category $F u n_{R}^{+}(c)$ are the Yoneda functors, that is $Y_{V}=\operatorname{Hom}_{R H c}(-, V)$, where $V$ is a finitely generated $p$-permutation $R H c$-module. Let $\left(F_{\bullet}, \eta_{\bullet}\right)$ be a right bounded complex of Yoneda functors. That is the nonzero terms are of the form $F_{i}=H_{R m_{R}}\left(-, V_{i}\right)$ for a finitely generated $p$-permutation $R H c$-module $V_{i}$. Let $M$ be a $p$-permutation $R G b$-module. Then, we have:

$$
\begin{aligned}
\left(L_{X_{\bullet}}\left(F_{\bullet}\right)\right)_{k}(M) & =\bigoplus_{i-j=k} F_{i}\left(X_{j} \otimes M\right) \\
& =\bigoplus_{i-j=k} \operatorname{Hom}_{R H}\left(X_{j} \otimes_{R G} M, V_{i}\right) \\
& \cong \bigoplus_{i-j=k} \operatorname{Hom}_{R G}\left(M, \operatorname{Hom}_{R H}\left(X_{j}, V_{i}\right)\right) .
\end{aligned}
$$

Since $X_{j}$ is projective as $R H c$-module, we have, by Corollary 9.4.2 [8], an isomorphism of functors

$$
\operatorname{Hom}_{R H c}\left(X_{j},-\right) \cong \operatorname{Hom}_{R}\left(X_{j}, R\right) \otimes_{R H c}-.
$$

Now, $\operatorname{Hom}_{R}\left(X_{j}, R\right)$ is a permeable bimodule. Then the $R G b$-module

$$
\operatorname{Hom}_{R H}\left(X_{j}, V_{i}\right) \cong \operatorname{Hom}_{R}\left(X_{j}, R\right) \otimes_{R H c} V_{j} .
$$

is a $p$-permutation $R G b$-module.

Since the isomorphism $\operatorname{Hom}_{R H}\left(X_{j} \otimes_{R G} M, V_{i}\right) \cong \operatorname{Hom}_{R G}\left(M, \operatorname{Hom}_{R H}\left(X_{j}, V_{i}\right)\right)$ is natural in $M$, we have an isomorphism of functors

$$
\left(L_{X_{\bullet}}\left(F_{\bullet}\right)\right)_{k} \cong \bigoplus_{i-j=k} \operatorname{Hom}_{R G}\left(-, \operatorname{Hom}_{R H}\left(X_{j}, V_{i}\right)\right) .
$$

So $L_{X_{\bullet}}\left(F_{\bullet}\right)_{k}$ is a (finite) direct sum of finitely generated projective functors.

Theorem 5.8 Let $G$ and $H$ be two finite groups, let $b$ be a block of $R G$ and $c$ be a block of $R H$. If the block algebras $R G b$ and $R H c$ are permeable derived equivalent, then the

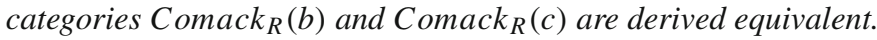

Proof It is enough to check that $F_{u n}^{+}(b)$ and $F u n_{R}^{+}(c)$ are derived equivalent. Let $X$ be a permeable tilting complex for $R H c$ and $R G b$. There exists a contractible complex of permeable $R H c$-bimodules such that:

$$
X \otimes_{R G b} X^{*}=R H c \oplus C,
$$

Then as functors between the homotopy category $K^{-}\left(\operatorname{proj}\left(F u n_{R}^{+}(c)\right)\right)$, we have:

$$
\begin{aligned}
L_{X^{*}} \circ L_{X} & \cong L_{X \otimes_{R G b} X^{*}} \\
& \cong L_{R H c \oplus C} \\
& \cong L_{R H c} \oplus L_{C} \\
& \cong L_{R H c} .
\end{aligned}
$$

Now, it is clear that $L_{R H c}$ is the identity of $K^{-}\left(\operatorname{proj}\left(F u n_{R}^{+}(c)\right)\right)$. Similarly, we have:

$$
L_{X} \circ L_{X^{*}} \cong L_{R G b} .
$$

So the homotopy categories $K^{-}\left(\operatorname{proj}\left(F u n_{R}^{+}(c)\right)\right)$ and $K^{-}\left(\operatorname{proj}\left(F u n_{R}^{+}(b)\right)\right)$ are equivalent (as triangulated categories). By Theorem 6.4 [13], the categories $D^{b}\left(\operatorname{Comack}_{R}(c)\right)$ and $D^{b}\left(\operatorname{Comack}_{R}(b)\right)$ are equivalent. 


\section{Applications}

\subsection{Nilpotent blocks}

Although the determinant of the Cartan Matrix of a block $b$ of $k G$ is a power of $p$, for the corresponding blocks of the Mackey algebra, it is much more complicated (see [6]). By the results of Section 7 of [16] this determinant is non zero. However the determinant of the Cartan matrix of a block of a cohomological Mackey algebra can be zero. In Theorem 4.2 of [6] Bouc proved that the Cartan matrix of $\operatorname{co\mu }_{k}(b)$ is non singular if and only if the block $b$ is a nilpotent block with cyclic defect group. This proof is based on a combinatorial approach, and it may be surprising that nilpotent blocks and cyclic defect groups appear in that situation. We will apply Theorem 5.8 to this situation, and show that it is in fact very natural.

Throughout this subsection $k$ denotes an algebraically closed field of characteristic $p>0$. Let $B$ be a block of $k G$, for an arbitrary finite group $G$. If $B$ is a nilpotent block with defect group $P$, then by Puig's Theorem (see [12] or [9]), there is an isomorphism of $k$-algebras,

$$
B \cong \operatorname{Mat}(m, k P),
$$

for some $m \in \mathbb{N}$. For the cohomological Mackey algebras, we can lift a Morita equivalence between blocks of group algebras, but for this we need that the equivalence sends $p$-permutation modules to $p$-permutation modules. Unfortunately it is not always the case. If the reader is not convinced by this fact she/he might look at Section 6.2 of this paper, or at Section 7.4 of [14].

By the results of sections 7.3 and 7.4 of [14] and results of [4] and [11], if $p>2$, or $P$ is abelian (N.B. in fact one can ask weaker condition in case of $p=2$ ), we can replace the bimodule which gives the Morita equivalence between $B$ and $k P$ by a splendid tilting complex of $B-k P$-bimodules.

Corollary 6.1 Let $B=k G b$ be a nilpotent block with defect $p$-group $P$. If $p=2$ assume that $P$ is abelian. Then

$$
D^{b}\left(\operatorname{co\mu }_{k}(G) \iota(b)-M o d\right) \cong D^{b}\left(\operatorname{co\mu }_{k}(P)-M o d\right) \text { as triangulated categories. }
$$

Since the determinant of Cartan matrices is invariant under derived equivalences, the determinant of the Cartan matrix $\operatorname{co\mu }_{k}(G) \iota(b)$ is non zero if and only if the determinant of the Cartan matrix $\cos _{k}(P)$ is non zero. However it is well known that this is the case if and only if the group $P$ is cyclic: indeed the projective indecomposable cohomological Mackey functors for a $p$-group $P$ are $F P_{I n d_{Q}^{P}(k)}$ for $Q \leqslant P$. By adjunction, the coefficient of the Cartan matrix indexed by two projective $F P_{I n d_{Q}^{P}(k)}$ and $F P_{I n d_{Q^{\prime}}^{P}(k)}$ is:

$$
\begin{aligned}
C_{Q, Q^{\prime}} & =\operatorname{dim}_{k} \operatorname{Hom}_{k P}\left(\operatorname{Ind}_{Q}^{P}(k), \operatorname{Ind}_{Q^{\prime}}^{P}(k)\right) \\
& =\operatorname{dim}_{k} \operatorname{Hom}_{k P}\left(k, \operatorname{Res}_{Q}^{P} \operatorname{Ind}_{Q^{\prime}}^{P} k\right) \\
& =\operatorname{Card}\left(\left[Q \backslash P / Q^{\prime}\right]\right) .
\end{aligned}
$$

By the main result of [15], this matrix is non degenerate if and only if $P$ is cyclic.

6.2 Application to the representation theory of finite groups

As immediate, but useful corollary of Proposition 4.5, we have: 
Corollary 6.2 Let $G$ and $H$ be two finite groups. Let $b$ be a block of $R G$ and $c$ be a block of $R H$. If the cohomological Mackey algebras $\operatorname{co\mu }_{R}(G) \iota(b)$ and $\operatorname{co\mu }_{R}(H) \iota(c)$ do not have the same Cartan matrix, then RGb and RHc are not 'splendidly' Morita equivalent.

This is useful since there are algorithms which compute these Cartan matrices. By testing this algorithm, the author found an astonishing (at least for him) example of nilpotent blocks with quaternion defect group, where the behavior of the simple modules involves rather sophisticated properties of number theory.

For this subsection $k$ will be an algebraically closed field of characteristic 2 . Let $p$ be an odd prime. Let $X_{p^{3}}$ be an extra-special group of exponent $p$, that is:

$$
X_{p^{3}}=<a, b, z ; a^{p}=b^{p}=z^{p}=1,[a, b]=z,[a, z]=[b, z]=1>.
$$

Let $Q_{8}$ be a quaternion group of order 8 , that is:

$$
Q_{8}:=<i, j ; i^{4}=1, i^{2}=j^{2}, j i j^{-1}=i^{-1}>
$$

Then, one can represent $Q_{8}$ as a subgroup of $G L_{2}\left(\mathbb{F}_{p}\right)$ by sending $i$ to the matrix $\left(\begin{array}{cc}0 & -1 \\ 1 & 0\end{array}\right)$ and $j$ to the matrix $\left(\begin{array}{cc}x & y \\ y & -x\end{array}\right)$, where $x^{2}+y^{2}=-1$. Such $x, y \in \mathbb{F}_{p}$ always exist.

A matrix $\left(\begin{array}{ll}\alpha & \beta \\ \gamma & \delta\end{array}\right)$ induces an automorphism of $X_{p^{3}}$ defined by:

- $a \mapsto a^{\alpha} b^{\beta}$

- $b \mapsto a^{\gamma} b^{\delta}$

- $z \mapsto z^{\alpha \delta-\beta \gamma}$.

Let us consider $G=X_{p^{3}} \rtimes Q_{8}$, where $Q_{8}$ acts on $X_{p^{3}}$ via its representation in $G L_{2}\left(\mathbb{F}_{p}\right)$.

Lemma 6.3 Let $k$ be an algebraically closed field of characteristic 2. There are $\frac{p^{2}-1}{8}+p$ blocks of $k G$.

- $\frac{p^{2}-1}{8}$ blocks with defect 0 .

- $p$ nilpotent blocks with $Q_{8}$ as defect group.

Sketch of proof. Since $X_{p^{3}}$ is a $2^{\prime}$-group, the blocks of this group are in bijection with the isomorphism classes of simple modules. There are $p^{2}-1$ representations which factorize through $C_{p} \times C_{p}=X_{p^{3}} / D\left(X_{p^{3}}\right)$. By usual clifford theory there are $\frac{p^{2}-1}{8}$ blocks of $k G$ covering all these blocks. Now there are $p-1$ blocks of $k X_{p^{3}}$ corresponding to the simple modules of dimension $p$, induced by a character of $k C_{p}$. Let $\zeta$ be a primitive $p$-root of 1 in $k$, then the simple module $V_{\zeta}$ of dimension $p$ is:

$$
V_{\zeta}=\operatorname{Ind}_{<a, z>}^{X_{p^{3}}} \operatorname{Inf} f_{<z>}^{<a, z>} k_{\zeta} .
$$

Here $k_{\zeta}$ is the irreducible $k<z>$-module of dimension 1 induced by $\zeta$. The inertie group of $V_{\zeta}$ is $G$, so this gives a simple module of $k G$ with $Q_{8}$ as vertex. We denote by $L_{\zeta}$ the $k G$-module such that $\operatorname{Res}_{X_{p^{3}}}^{G} L_{\zeta} \cong V_{\zeta}$.

Proposition 6.4 Let $\zeta$ be a p-root of 1 in $k$. Let $L_{\zeta}$ be the corresponding simple $k G$-module and let $b_{\zeta}$ be the corresponding block. Then

- If $p \not \equiv 1 \bmod 8$, then $k G b_{\zeta}$ is not splendidly Morita equivalent to $k Q_{8}$. 
- Let $p \equiv 1 \bmod 8$ and let $t(\zeta)=\sum_{x \in I} \zeta^{x}$, where I is the set of quadratic residues mod p. If $t(\zeta)=0$, then $k G b_{\zeta}$ is splendidly Morita equivalent to $k Q_{8}$.

- If $t(\zeta)=1$ and $p=17$, then $k G b_{\zeta}$ is not splendidly Morita equivalent to $k Q_{8}$.

Remark 6.5 The condition $p=17$ appears only because we are not able to find a general proof of this result. However it seems that the result should be true for all $p \equiv 1 \bmod 8$. In particular we check it with GAP in several cases.

Proof Here, we are very sketchy. The first part follows from Mazza's work. See Section 4.2 of [10]. It is shown that $\operatorname{Res}_{Q_{8}}^{G} L_{\zeta}$ is an endo-trivial module with source $S_{\zeta}$ such that $\operatorname{dim}_{k} S_{\zeta} \equiv p \bmod 8$. So if $p \not \equiv 1 \bmod 8$, then $L_{\zeta}$ is not a 2-permutation module and the Morita equivalence is not splendid.

If $p \equiv 1 \bmod 8$, then the source can be either the trivial module or an endo-trivial module of dimension 9. Let $w \in \mathbb{F}_{p}$ such that $w^{2}=1$ and let $b$ be a generators of $\mathbb{F}_{p}^{\times}$. The module $\operatorname{Res}{ }_{Q_{8}}^{G} L_{\zeta}$ is a trivial source module if and only if $k$ is a direct summand of $\operatorname{Res}_{Q_{8}}^{G} L_{\zeta}$. This happens if and only if there is a vector $v \in L_{\zeta}^{Q_{8}}$ and an invariant linear form $\phi$ on $\operatorname{Res}_{Q_{8}}^{G} L_{\zeta}$ such that $\phi(v)=1$.

Let $i \in \mathbb{F}_{p}^{\times} /<w>$. Let $t_{i}:=\zeta^{b^{i}}+\zeta^{w b^{i}}+\zeta^{w^{2} b^{i}}+\zeta^{w^{3} b^{i}} \in \mathbb{F}_{2^{\frac{p-1}{8}}}$ be a Gaussian sum. Let $M$ be the matrix indexed by $\mathbb{F}_{p}^{\times} /<w>$, where the $(i, j)$ th. coefficient is $t_{i+j}$. One can check that $L_{\zeta}$ is a 2-permutation module if and only if the constant vector $(1,1, \ldots, 1)^{t}$ is in the image of $M-I d$.

Now, if $t(\zeta)=0$, we have $(M-I d) \cdot(1,0,1,0, \cdots 1,0)^{t}=(1,1, \ldots, 1)^{t}$.

If $p=17$ and $t(\zeta)=1$ an easy computation shows that $(1,1, \ldots, 1)$ canot be in the image of $M-I d$.

Acknowledgments This work is part of my $\mathrm{PhD}$ at Université de Picardie Jules Verne supported by a BDICNRS-FEDER Grant. I would like to thank Serge Bouc, my PhD advisor for many helpful conversations. I am very grateful to the referee for the careful reading of this paper. In particular for pointing out a repeated mistake. I finally thank ECOS-CONACYT for the financial support in the project M10M01.

\section{References}

1. Artin, M., Grothendieck, A., Verdier, J.: SGA 1963-1964. Théorie des topos et cohomologie étale des schémas, vol. 1. Springer, Berlin (1972)

2. Benson, D.: Representations and Cohomology: Basic Representation Theory of Finite Groups and Associative Algebras, vol. I. Cambridge University Press, Cambridge (1995)

3. Bouc, S.: Green Functors and $G$-sets, volume 1671 of Lecture Notes in Mathematics. Springer, Berlin (1997)

4. Bouc, S.: The Dade group of a p-group. Invent. Math. 164(1), 189-231 (2006)

5. Bouc, S.: Complexity and cohomology of cohomological Mackey functors. Adv. Math. 221(3), 983-1045 (2009)

6. Bouc, S.: On the Cartan matrix of Mackey algebras. Trans. Am. Math. Soc. 363(8), 4383-4399 (2013)

7. Green, J.A.: Axiomatic representation theory for finite groups. J. Pure Appl. Algebra 1(1), 41-77 (1971)

8. König, S., Zimmermann, A.: Derived Equivalences for Group Rings. Lecture Notes in Mathematics. Springer, Berlin (1998)

9. Külshammer, B.: Nilpotent blocks revisited. In Groups, rings and group rings, volume 248 of Lecture Notes in Pure and Applied Mathematics, pp. 263-274. Chapman \& Hall/CRC, Boca Raton, FL (2006)

10. Mazza, N.: Endo-permutation modules as sources of simple modules. J. Group Theory 6(4), 477-497 (2003)

11. Mazza, N.: Modules d'endo-permutation. PhD thesis, Faculté des Sciences de l'université de Lausanne (2003)

12. Puig, L.: Nilpotent blocks and their source algebras. Invent. Math. 93(1), 77-116 (1988) 
13. Rickard, J.: Morita theory for derived categories. J. Lond. Math. Soc. 39, 436-456 (1989)

14. Rickard, J.: Splendid equivalences: derived categories and permutation modules. Proc. Lond. Math. Soc. 72, 331-358 (1996)

15. Thévenaz, J.: A characterization of cyclic groups. Archiv der Mathematik 52(3), 209-211 (1989)

16. Thévenaz, J., Webb, P.: The structure of Mackey functors. Trans. Am. Math. Soc. 347(6), 1865-1961 (1995)

17. Weibel, C.A.: An Introduction to Homological Algebra. Cambridge University Press, Cambridge (1994)

18. Yoshida, T.: On G-functors (ii): Hecke operators and G-functors. J. Math. Soc. Jpn. 35, 179-190 (1983) 